\begin{tabular}{|c|l|}
\hline Title & Facet bending in the driven crystall line curvature flow in the plane \\
\hline Author(s) & Giga, Y oshikazu; Rybka, Piotr \\
\hline Citation & Hokkaido University Preprint Series in Mathematics, 763, 1-34 \\
\hline Issue Date & 2005-12-28 \\
\hline DOI & 10.14943/83913 \\
\hline Doc URL & http://hdl.handle.net/2115/69571 \\
\hline Type & bulletin (article) \\
\hline File Information & pre763.pdf \\
\hline
\end{tabular}

Instructions for use 


\title{
Facet bending in the driven crystalline curvature flow in the plane
}

\author{
Yoshikazu Giga $^{1}$, Piotr Rybka ${ }^{2}$ \\ ${ }^{1}$ Graduate School of Mathematical Sciences \\ University of Tokyo \\ Komaba 3-8-1, Tokyo 153-8914, Japan \\ e-mail: labgiga@ms.u-tokyo.ac.jp \\ ${ }^{2}$ Institute of Applied Mathematics and Mechanics, Warsaw University \\ ul. Banacha 2, 07-097 Warsaw, Poland \\ fax: +(48 22) 554 4300, e-mail: rybka@mimuw. edu.pl
}

December 28, 2005

\begin{abstract}
We study simple cases of crystalline driven curvature flow with spatially nonuniform driving force term. We assume special monotonicity properties of the driving term, which are motivated by our previous work on Berg's effect. We show that initially flat facets, if loosely speaking they are to large, then they begin to bend.
\end{abstract}

Key words: singular energies, bending of facets, driven curvature flow, variational principle

2000 Mathematics Subject Classification. Primary: 53C44 Secondary: 35K55

\section{Introduction}

We are concerned with an anisotropic curvature flow equation with a driving force term $\sigma$ of the form

$$
\sigma=\operatorname{div}_{S} \xi+\beta V \quad \text { on } \Gamma(t),
$$

where $\Gamma(t)$ is an evolving curve in the plane. Here $V$ denotes the normal velocity in the direction of the normal vector field $\mathbf{n}$ and $\beta$ is a kinetic coefficient depending on $\mathbf{n}$ which is assumed to be positive. The term $-\operatorname{div}_{S} \xi$ denotes the anisotropic curvature. It is defined by using an interfacial energy density $\gamma$ which is given, convex and positively homogeneous of degree one in $\mathbb{R}^{2}$. Formally, $\xi$ is defined by

$$
\xi=\nabla \gamma(\mathbf{n})
$$


If $\gamma(p)=|p|$, then $\xi=\mathbf{n}$ so its surface divergence term $-\operatorname{div}_{S} \xi$ is nothing but the Euclidean curvature. In this paper we are interested in the case when $\gamma$ is not $C^{1}$, for example $\gamma$ is crystalline, i.e. $\gamma$ is piecewise linear. Then the meaning of (1.2) is not clear since $\nabla \gamma(\mathbf{n})$ is nowhere well-defined. Instead, we consider

$$
\xi(x) \in \partial \gamma(\mathbf{n}(x)) \quad \text { on } \Gamma(t),
$$

where $\partial$ denotes the subdifferential of a convex function $\gamma$. The vector field $\xi$ is often called a Cahn-Hoffman vector.

Our eventual goal is to find a solution $\Gamma(t)$ satisfying (1.1), (1.3) with a given initial data $\Gamma(0)$, where $\gamma$ is not $C^{1}$, in particular $\gamma$ may be crystalline, when $\sigma$ is given. Such a problem was first proposed by J. Taylor, [Ta1], for the case $\sigma \equiv 0, \beta \gamma=$ const, $\gamma$ crystalline and independently by S. Angenent and M. Gurtin, [AG], in a general form. In the latter case when $\sigma$ is independent of the spatial variables, the unique solvability of the initial value problems has been established in various settings. The reader is referred to papers [GG3], [GG5] by M.-H. Giga and Y. Giga (where a level-set solution is constructed for arbitrary initial data) and papers cited there. A key observation is that a flat portion of $\Gamma(t)$ (called a facet) stays flat when $\gamma$ is a crystalline [AG], [Ta1].

However, if $\sigma$ depends on the spatial variable $x=\left(x_{1}, x_{2}\right)$, such property may not hold. In fact, as already observed in [GG1], it actually happens that there is no $\xi$ satisfying (1.1) and (1.3) with constant $V$ on a facet. In [GG1], $\Gamma(t)$ is assumed to be a graph-like function and $\beta$ is taken so that $\beta V$ is an upward velocity. An explicit facet-bending solution is constructed when $\sigma$ is a piecewise linear function in $x$. If $\sigma$ is close to a constant and a facet is short, then this facet may persist. In fact, there is a comparison principle [BGN] for a special situation, but in general if one imposes facet stability (without (1.3) but assuming that $-\operatorname{div}_{S} \xi+\sigma$ equals the crystalline curvature plus the average of $\sigma$ over a facet which is weaker than (1.3)) then the comparison principle may not hold (see [GG2] by M.-H. Giga and Y. Giga). The solvability of initial value problem for spatially dependent $\sigma$ is known only for very restrictive settings [GG1], [GG4] like graph-like functions so that it can be reduced to the theory of nonlinear semigroups initiated by Y. Kōmura. An explicit form of a solution is only given when $\sigma$ is piecewise linear.

Our goal in this paper is to understand the explicit shape of the evolution of $\Gamma(t)$ satisfying (1.1), (1.3) after the onset of the bending of facets when $\sigma$ is not necessarily (piecewise) linear in spatial variables but still special. We consider the case that the interfacial energy is of the form

$$
\gamma\left(p_{1}, p_{2}\right)=\left|p_{1}\right| \gamma_{\Lambda}+\left|p_{2}\right| \gamma_{T},
$$

with two positive constants $\gamma_{\Lambda}$ and $\gamma_{T}$. Its Wulff shape $W_{\gamma}$ equals

$$
W_{\gamma}=\bigcap_{|m|=1}\left\{x \in \mathbb{R}^{2}: m \cdot x \leq \gamma(m)\right\}
$$

and it is a rectangle

$$
\left\{\left(x_{1}, x_{2}\right) \in \mathbb{R}^{2}:\left|x_{1}\right| \leq \gamma_{\Lambda},\left|x_{2}\right| \leq \gamma_{T}\right\} .
$$

To simplify the problem we further assume that

$$
\beta\left(n_{1}, n_{2}\right)=\frac{1}{\max \left(\left|n_{1}\right|,\left|n_{2}\right|\right)},
$$


where $n_{1}^{2}+n_{2}^{2}=1$, so that $\beta V$ equals upward velocity for $n_{2} \geq\left|n_{1}\right|$ and velocity in the direction of $x_{1}$ when $0 \leq n_{1} \leq\left|n_{2}\right|$ where $\mathbf{n}=\left(n_{1}, n_{2}\right)$ is an outer normal of $\Gamma(t)$.

Our assumptions on $\sigma$ may be expressed by saying that it grows from the center of facets to the edge of the facet. This causes facet bending. To simplify the problem we further assume some symmetry properties of $\sigma$ related to $\Gamma$.

We consider two situations as follows.

(i) $\Gamma(t)$ is a graph of a function with Neumann boundary condition; in particular $\Gamma(0)$ may be a segment of a straight line, $[-L, L] \times\{0\}$. We assume that $\sigma=\sigma\left(x_{1}\right)$ is of class $C^{1}$ and $\sigma_{x_{1}}>0$ for $x_{1}>0$ and $\sigma\left(-x_{1}\right)=\sigma\left(x_{1}\right)$ i.e. $\sigma$ is even.

(ii) $\Gamma(0)$ is typically a rectangle symmetric with respect to $x_{1}$ and $x_{2}$ axes. The function $\sigma=\sigma\left(t, x_{1}, x_{2}\right)$ is even in $x_{1}$ and $x_{2}$ and $\sigma_{x_{i}}>0$ for $x_{i}>0$.

We shall construct a solution of (1.1), (1.3) locally-in-time rather explicitly in the sense that its profile is easy to understand. In the case (i) our solution has three facets: one is near 0 , the other two are near $x_{1}= \pm L$. The curve $\Gamma(t)$ is given by the graph of $d\left(x_{1}, t\right)(t \geq 0)$ which is nondecreasing in $x_{1}$ for $x_{1}>0$ and even in $x_{1}$. So curved parts are possible. Of course, our $\sigma$ must violate the stability condition (facet preserving property studied in [GR3], [GR4]) forcing the initial facet to break. A solution is constructed by taking minimal section of $\xi$ which is consistent with nonlinear semigroup solution. We say the pair $(\Gamma, \xi)$ is a variational solution of (1.1), (1.3) if in addition $\xi$ satisfies a minimality condition (see Section 2). Similar results are established for the case (ii). As far as the uniqueness is concerned we are able to prove that the variational solution is unique for (i) and (ii). In the case of (i) uniqueness holds even among more general solutions so that solution must be variational. This point will be explained in the uniqueness theorem in Section 2. The origin of such approach goes back to [FG] or [GG1].

Our analysis permits us to have a close look at the properties of solutions. A key observation is that the Cahn-Hoffman vector satisfies the condition $\partial \xi / \partial \tau=0$ at the bending points of $\Gamma(0)$, where $\tau$ is a unit tangent to $\Gamma(0)$. We say this condition is the tangency condition. We show that it is propagated in time by the variational solutions, (this notion will be introduced in Section 2). That is, such identity must occur at the intersection points of a facet and a curved part. This observation is important for construction of solutions to the problem.

Another observation is that there may be no region where $\Gamma$ is flat inside curved parts. In the case of (i) this can be explained that $d_{x_{1}}>0$ must hold for curved parts. If an initially flat region cannot stay flat, then it gets immediately curved. This will be explained in subsection 2.2.

This paper is a continuation of of our earlier work, see [GR1], [GR2], [GR3], [GR4], where the evolution system consisted of (1.1) coupled to a (quasi-steady approximation to) diffusion equation. These papers were devoted to finding regions of parameters guaranteeing facet stability. Here, we investigate the onset of instability in a simplified setting being the introduction to the full problem.

We study two situations, in Section 2 in order to develop the main idea we consider the case of a graph evolving by the curvature flow. We also introduce the necessary notions, like matching condition and tangency condition. We define the notion of solution, which is subsequently narrowed down to the variational one. A crucial role here is played by a variational principle. We prove local in time existence of unique solutions to (1.1), it is worth stressing that uniqueness is obtained for slightly larger class than the variational 
solutions. Moreover, comparison principle holds since the problem can be approximated by usual uniform parabolic problem having a comparison principle.

In section 3 we develop the same notions for the (bent) rectangle. Here, the analysis is more involved and the preliminary analysis of $\S 2$ is the guiding principle for us. Here, we also show local in time existence of unique variational solutions to (1.1), i.e. the uniqueness result is less general than for graphs. We believe that a comparison principle must be valid for this problem but we do not pursue this point in this paper. When $\sigma$ is independent of $x$, a comparison principle has been proved in [GG3].

\section{Simple graph case}

In this section we present the main ideas, they will be further developed in the next section, to deal with a bending 'rectangle'. For the sake of clarity of exposition we consider in this section a very simple situation, which may be interpreted as a constrained growing crystal, which does not react with the container wall and with prescribed supersaturation field $\sigma$. Namely, we consider $\Gamma(t)$ (the crystal surface) being the graph of a function $d:[-L, L] \rightarrow$ $\mathbb{R}$, while the crystal occupies the region $\{(x, y): 0 \leq y \leq d(t, x), x \in[-L, L]\}$. Our goal is to study how the curve $\Gamma$ bends, when it gets too large. Thus, in order to develop intuition about what happens without introducing too much technical complications we make additional simplification.

\subsection{Preliminary analysis}

We present here our assumptions on the system. Namely, we fix the angle between $\Gamma(t)$ and the lines $\{|x|=L\}$, to be $\frac{\pi}{2}$. Furthermore, as we have already mentioned in the introduction $d$ is even.

We have indicated in (i) of $\S 1$ our assumptions on $\sigma$ necessary to proceed with our analysis. Namely, we consider $\sigma$ in $C^{1}([-L, L])$ satisfying

$$
\sigma(x)=\sigma(-x) \geq 0
$$

and

$$
\frac{d \sigma}{d x}(x)>0 \quad \text { for } x>0 .
$$

Let us mention that they are consistent with results proved in [GR2].

We also have to define $\gamma$ the energy density function, $\gamma: \mathbb{R}^{2} \rightarrow \mathbb{R}$. We consider $\gamma$ given by formula (1.4), i.e.

$$
\gamma\left(p_{1}, p_{2}\right)=\left|p_{1}\right| \gamma_{\Lambda}+\left|p_{2}\right| \gamma_{T}
$$

If we re-write (1.1) using the definition of the surface divergence (see e.g. [Si]) while taking into account that $\Gamma(t)$ is a graph we will obtain

$$
\beta\left(d_{x}\right) \frac{d_{t}}{\sqrt{1+d_{x}^{2}}}=\sigma-\tau \cdot \frac{\partial \xi}{\partial \tau}
$$

where $\tau$ is a unit tangent and $\mathbf{n}$ is a outer normal vector to $\Gamma(t)$. In our setting these two vector fields are defined only $\mathcal{H}^{1}$-a.e. Whenever convenient, we use the subscript $t$ and $x$ 
to denote partial differentiation. In situation in which this may lead to confusion we will avoid this notation. We require not only

$$
\xi(t, x) \in \partial \gamma(\mathbf{n}(x)), \quad \text { for } \mathcal{H}^{1} \text {-a.e. } x \in \Gamma(t),
$$

but also

$$
\operatorname{div}_{S} \xi \in L^{2}(\Gamma(t)) .
$$

Thus, we come to the conclusion that

$$
\tau \cdot \frac{\partial \xi}{\partial \tau} \in L^{2}(\Gamma(t))
$$

The angle condition translates into

$$
\frac{\partial}{\partial x} d(t, x)=0 \quad \text { at } \quad x= \pm L .
$$

However, this condition is artificial because it does not take into account any possible interaction of the crystal with the container wall.

We introduce a notation for two special vectors, namely we shall write $\mathbf{n}_{\Lambda}=(0,1)$ and $\mathbf{n}_{R}=(1,0)$. The last one is a normal to the vertical boundary of the container. We also specify an additional requirement for $\xi$

$$
\left.\xi(p)\right|_{p=( \pm L, d(t, \pm L))} \in \partial \gamma\left(\mathbf{n}_{\Lambda}\right) \cap \partial \gamma\left( \pm \mathbf{n}_{R}\right)
$$

reflecting the assumption that the segments $\{ \pm L\} \times[0, d(t, \pm L)]$ are facets of our crystal, however they are artificially constrained to be motionless. We stress that by (2.4) or (2.5) our $\xi$ is sufficiently regular to take its trace at $x= \pm L$.

The evolution system (1.1) for $\Gamma=\operatorname{graph}(d)$ has to be augmented with initial data

$$
d(0, x)=d_{0}(x) .
$$

Function $d_{0}$ is not arbitrary, later we shall comment on its form.

\subsubsection{Definition of solution}

Let us introduce our notion of a solution to (1.1). We will not strive to make our present definition general, we just want to extend the notion of solutions to crystalline curvature flow (see [AG], [Ta2], [St], [R]) to accommodate the present case. Thus, in particular the angles at the vertexes are preserved. We stress that the crystalline curvature is also a global, but not pointwise concept. A discussion of the proper, general definition of solution is beyond the scope of this paper.

We mention here that a special role is played by a Cahn-Hoffman vector. Namely, a vector field $\xi: \Gamma \rightarrow \mathbb{R}^{2}$ is called a Cahn-Hoffman vector, if $\xi \in \mathcal{D}$, where

$$
\mathcal{D}=\left\{\xi \in L^{\infty}(\Gamma): \xi(x) \in \partial \gamma(\mathbf{n}(x)), \operatorname{div}_{S} \xi \in L^{2}(\Gamma),(2.7) \text { holds }\right\} .
$$

We are now ready for the definition of solution. We call by a solution to (1.1) a couple $(\Gamma, \xi)$ such that for some $T>0$ the following condition are satisfied:

(a) For each $t \in[0, T)$ the curve $\Gamma(t)$ is defined as the graph of a function $d$, i.e. $\Gamma(t)=\{(x, d(t, x)): x \in[-L, L]\}$. Moreover, $d$ is an element of $C([0, T) \times[-L, L])$, 
$d(\cdot, x)$ is Lipschitz continuous for each $x \in[-L, L]$ and for each $t \in[0, T)$ we have $d(t, \cdot) \in \operatorname{Lip}[-L, L]$

(b) $\xi: \bigcup_{t \in[0, T)}\{t\} \times \Gamma(t) \rightarrow \mathbb{R}^{2}$ is such that the function $t \mapsto \xi(t,(\cdot, d(t, \cdot)))$ is in $L^{\infty}\left([0, T) ; L^{2}(-L, L)\right)$ and for each $t \in[0, T)$ the field $\xi(t, \cdot)$ is a Cahn-Hoffman vector, and

(c) Equation (1.1) is satisfied in the $L^{2}$ sense for a.e. $t \geq 0$.

Let us mention that the time Lipschitz continuity (instead of $C^{1}$ property) is necessary to conduct the analysis in subsections 2.3 and 2.4. In 2.2 the function $d(\cdot, x)$ is in $C^{1}([0, T))$.

The present notion of solution may seem too broad, because it is not clear at all if it permits us to prove uniqueness. A rational for this doubt is we should close the system with a rule for selecting $\xi$. An explicit formula for $\xi$ is not always neither possible nor necessary. We rely here on a variational principle for selecting $\xi$. This approach is consistent with our previous work [GR3], [GR4], but also it is in the line of the work of Bellettini, Novaga and Paolini (see [BNP1]-[BNP3]).

\subsubsection{The variational principle}

We postulate that at each time instant $t$ the Cahn-Hoffman vector field $\xi(t, \cdot): \Gamma \rightarrow \mathbb{R}^{2}$ is a minimizer of

$$
\mathcal{E}(\xi)=\frac{1}{2} \int_{\Gamma(t)}\left|\sigma-\operatorname{div}_{S} \xi\right|^{2} d \mathcal{H}^{1}
$$

over $\mathcal{D}$. This choice of $\mathcal{E}$ is justified by the fact that the Euler-Lagrange equations for $\mathcal{E}$ over any flat facet (with the normal $\mathbf{n}_{\Lambda}$ ) is just

$$
\sigma-\operatorname{div}_{S} \xi=\text { const. }
$$

Let us also notice that the constant appearing here equals to $\beta V$.

On the other hand, on curved parts of $\Gamma$, i.e. where $\mathbf{n}$ is different from $\mathbf{n}_{\Lambda}$ and $\mathbf{n}_{R}$, the minimization is trivial, i.e. over one element. It is so because for such $\mathbf{n}$ the subdifferential $\partial \gamma(\mathbf{n})$ is a singleton.

We will say that a couple $(\Gamma, \xi)$ is a variational solution to (1.1) if it is a solution to (1.1) and in addition $\xi$ satisfies

(d) for each $t \geq 0 \xi(t, \cdot)$ is a solution to

$$
\mathcal{E}(\xi)=\min \{\mathcal{E}(\zeta): \zeta \in \mathcal{D}\}
$$

Some comments are in order. Namely, in case of $\Gamma$ being a line segment our minimization problem is in general of obstacle type, hence Euler-Lagrange equations (2.10) may not be satisfied a.e. This will have quite important implications, we will develop it below, as it seems to be the most interesting.

We stress that since $d$ is assumed to be Lipschitz continuous (but with some restriction, see (2.15) below) the normal vector $\mathbf{n}$ is defined only $\mathcal{H}^{1}$-a.e. In general we should not expect higher regularity of $d$.

We are not interested in studying a general graph motion under driven crystalline flow but rather the situation which arises past the phenomenon of bending of flat faces, i.e. past their loss of stability. The papers [GR3], [GR4] describe the evolution of (1.1) coupled to a diffusion field, where the shape of interface $\Gamma$ is persistent up to the point where the condition necessary for stability fails, hence the motivation for the present work. 
This is the reason for considering only a special type of initial conditions,

$$
\begin{array}{ll}
d(0, x)=R_{00} & \text { for } x \in\left[-\lambda_{0}, \lambda_{0}\right] \\
d(0, x)=R_{10} & \text { for } x \in\left[-L,-\lambda_{1}\right] \cup\left[\lambda_{1}, L\right] \\
d(0, \cdot)=d_{0}(\cdot) & \text { is increasing on }[0, L] .
\end{array}
$$

We shall see that these are the consequence of monotonicity of $\sigma$ over $(0, L)$. A particular case of these data is $d_{0} \equiv R_{00}$.

In order to deal with flat parts of $\Gamma$ we introduce a number of auxiliary notions. Here is the first one. Let us consider an open line segment $I$ in the plane, i.e. $I=(a, b) \equiv\{x=$ $a t+b(1-t), t \in(0,1)\}$, where $a, b \in \mathbb{R}^{2}$. We shall say that $I \subset \Gamma$, having normal equal to $\mathbf{n}_{\Lambda}$, is a faceted region of $\Gamma$ if is maximal (with respect to inclusion) and it satisfies

$$
\left.\left(\sigma-\operatorname{div}_{S} \xi\right)\right|_{I}=\text { const. }
$$

where $\xi$ is a solution to (2.11).

However, $\Gamma(t)$ is a graph, i.e. the image of segment $[-L, L]$ under the function

$$
x \mapsto(x, d(t, x))=: \tilde{d}(t, x) .
$$

Frequently it is more convenient to work with the inverse image of a faceted region $I$, $(\alpha, \beta)=\tilde{d}^{-1}(I)$. We stress that this definition permits $\Gamma(t)$ being a line segment which has more than one faceted region.

Subsequently, we will consider $\Gamma(t)$ such that $\left(-L,-l_{1}\right),\left(-l_{0}, l_{0}\right)$ and $\left(l_{1}, L\right)$ are the only inverse images of faceted regions of $\Gamma(t)$. Of course their endpoints $l_{0}, l_{1}$ may be functions of time. Initially we will consider $l_{0}<l_{1}$, but later we will also study the case $l_{0}=l_{1}$, i.e. of a flat facet which is going to break up.

Summarizing, we should distinguish two cases of exactly three faceted regions: (a) $\Gamma$ is a graph of a non-constant (increasing for $x>0$ ) function $d$ having exactly three faceted regions; (b) $\Gamma$ is a single line segment having three faceted regions.

We stress that (b) occurs at the onset of facet instability. In all cases $l_{0}, l_{1}$ are determined through the minimization problem (2.11). In case (a) it may happen that $l_{0}<\lambda_{0}$ as well as $l_{1}>\lambda_{1}$.

Proposition 2.1 Let us define

$$
E_{Z}=\left\{x \in\left(-l_{1},-l_{0}\right) \cup\left(l_{0}, l_{1}\right): \frac{\partial d}{\partial x}=0 \text { or } d \text { is not differentiable at } x\right\} .
$$

Let us suppose that $d$ satisfy (2.12), $\xi$ is a solution to (2.11) and (a) or (b) above hold. In addition we assume that

$$
\mathcal{H}^{1}\left(E_{Z} \backslash \text { int } E_{Z}\right)=0,
$$

where int $E_{Z}$ denotes the interior of $E_{Z}$. Then, $\xi$ is constant over $\left(-l_{1},-l_{0}\right) \cup\left(l_{0}, l_{1}\right)$.

Proof. (a) We assume that $d$ is not constant. Since $d$ is Lipschitz continuous and increasing on $\left(l_{0}, l_{1}\right)$, then $\tau$ the tangent vector to $\Gamma$ may have only jump discontinuities. Nonetheless, if we write $\tau=\left(\tau_{1}, \tau_{2}\right)$, then $\tau_{1}>0$ and $\tau_{2} \geq 0$. Hence, at no point on $\Gamma$ the normal equals to $-\mathbf{n}_{R}$. This and the formula for $\gamma$, see (1.4) imply that whenever $\tau_{2}>0$, i.e. $d_{x}>0$, then $\nabla \gamma(\mathbf{n}(x))$ is equal to $\left(-\gamma_{\Lambda}, \gamma_{T}\right)$. Because by assumption $d$ is Lipschitz 
continuous it follows that $\tau \cdot \mathbf{e}_{1} \geq a>0$. Hence $\tau \cdot \frac{\partial \xi}{\partial \tau}$, which is defined a.e. equals to $\left(\tau \cdot \mathbf{e}_{1}\right)^{2} \frac{\partial \xi}{\partial x}$, thus $\frac{\partial \xi}{\partial x} \in L^{2}[-L, L]$. In particular, this implies that $x \mapsto \xi(x, d(t, x))$ is continuous.

Let us consider int $E_{Z}=\bigcup_{i=1}^{\infty} J_{i}$, where possibly some of the open intervals $J_{i}$ are empty. Since the Euler-Lagrange equation does not hold on (non-empty) $J_{i}$ it follows that $\xi$ belongs to the boundary of $\partial \gamma(\mathbf{n})$ on $J_{i}$, i.e. $\xi \in\left\{\left(-\gamma_{\Lambda}, \gamma_{T}\right),\left(\gamma_{\Lambda}, \gamma_{T}\right)\right\}$. We notice that since the change of $\xi$ over $E_{Z} \backslash$ int $E_{Z}$ is zero, i.e.

$$
\int_{E_{Z} \backslash \text { int } E_{Z}} \frac{\partial \xi}{\partial x} d x=0
$$

then it follows that $\xi$ cannot attain two different values on $\left(l_{0}, l_{1}\right)$, a similar conclusion holds on $\left(-l_{1},-l_{0}\right)$. However, we did not determine the value of the constant.

(b) In this case (2.11) is the obstacle problem. By assumption on faceted region the Euler-Lagrange equation (2.10) holds, while at the same time, this equation (2.10) fails on $\left(-l_{1},-l_{0}\right) \cup\left(l_{0}, l_{1}\right)$ because $\xi$, being a minimizer of $\mathcal{E}$, touches the boundary of the constraint, i.e. it belongs to the boundary of $\partial \gamma\left(\mathbf{n}_{\Lambda}(x)\right)$ for $x \in\left[-l_{1},-l_{0}\right] \cup\left[l_{0}, l_{1}\right]$. Moreover, $\xi_{2}$ is always here equal to $\gamma_{T} \equiv \gamma\left(\mathbf{n}_{R}\right)$. Since $d$ is constant, then $\operatorname{div}_{S} \xi$ on $[-L, l]$ takes the form $\frac{\partial \xi_{1}}{\partial x}$, hence $\xi_{1}$ is continuous. As a result, $\xi_{1}$ is constant over $\left(-l_{1},-l_{0}\right) \cup\left(l_{0}, l_{1}\right)$. However, the values of $\xi_{1}$ have to be determined yet.

We want to write down equations of motion in a form suitable for analysis while taking into account our notion of solution to (1.1). We note that continuity of $\xi$ and the symmetry of $\xi$ imply that $\xi_{1}(0)=0$. On inverse images of faceted regions $d$ is constant, hence the following definitions make sense:

$$
R_{0}(t):=\left.d(t, \cdot)\right|_{\left[0, l_{0}\right]}, \quad R_{1}(t):=\left.d(t, \cdot)\right|_{\left[l_{1}, L\right]} \cdot
$$

If at each $t>0$ condition (2.15) is satisfied and $\Gamma(t)$ has three faceted regions, then (1.1) (i.e. system (2.3)) takes on the following form, where due to the symmetry we can consider only $x>0$ :

$$
\begin{aligned}
\sigma-\frac{\partial \xi_{1}}{\partial x} & =\beta\left(\mathbf{n}_{\Lambda}\right) \dot{R}_{0} \quad \text { on } \quad\left[0, l_{0}\right] \\
\sigma-\frac{\partial \xi_{1}}{\partial x} & =\beta\left(\mathbf{n}_{\Lambda}\right) \dot{R}_{1} \quad \text { on } \quad\left[l_{1}, L\right] \\
\sigma & =\frac{\beta\left(d_{x}\right)}{\sqrt{1+d_{x}^{2}}} d_{t} \quad \text { on } \quad\left(l_{0}, l_{1}\right)
\end{aligned}
$$

In principle $l_{0}$ and $l_{1}$ are also functions of time and if so they are a part of the solution.

\subsubsection{The matching conditions}

At the moment equalities

$$
R_{0}(t)=d\left(t, l_{0}\right), \quad R_{1}(t)=d\left(t, l_{1}\right)
$$

are trivial consequences of continuity of $d(t, \cdot)$. However, if we try to solve (2.16), then we have to make sure that indeed (2.17) holds. For that reason we shall call (2.17) the matching conditions. 
In order to proceed we have to make assumptions on $\beta$. We are striving for a simple yet non-trivial setting. For these reasons in (1.5) we adopted $\beta\left(n_{1}, n_{2}\right)=\frac{1}{\max \left(\left|n_{1}\right|,\left|n_{2}\right|\right)}$. If $\Gamma$ is the graph of $d$, then $\mathbf{n}=\left(-d_{x}, 1\right) / \sqrt{1+d_{x}^{2}}$, for $\left|d_{x}\right| \leq 1$ we obtain

$$
\beta(\mathbf{n})=\sqrt{1+d_{x}^{2}}
$$

and particular

$$
\beta\left(\mathbf{n}_{\Lambda}\right)=1 .
$$

The restriction $\left|d_{x}\right| \leq 1$ means that (2.18) is valid for normals which form with the $x$ axis the angle between $\frac{\pi}{4}$ and $\frac{3}{4} \pi$.

Before we try to find $\xi$ we note a couple of simple statements and continue introducing new notions. The first one is stated for the sake of clarity.

Proposition 2.2 Let us assume that $d:[0, T) \times[0, L] \rightarrow \mathbb{R}$ is a function with the following properties:

(a) for each $x \in[0, L]$ the function $d(\cdot, x)$ is in $C^{1}([0, T))$;

(b) there is $x_{0} \in(0, L)$ such that $d(t, \cdot) \in C^{1}\left(\left[0, x_{0}\right]\right)$ and $d(t, x) \equiv R(t)$ for $x>x_{0}$; If $d(0, \cdot) \in C([0, L])$, then $d(t, \cdot) \in C([0, L])$ if and only if $\frac{\partial d}{\partial t}\left(t, x_{0}\right)=\dot{R}(t)$.

An obvious proof is omitted.

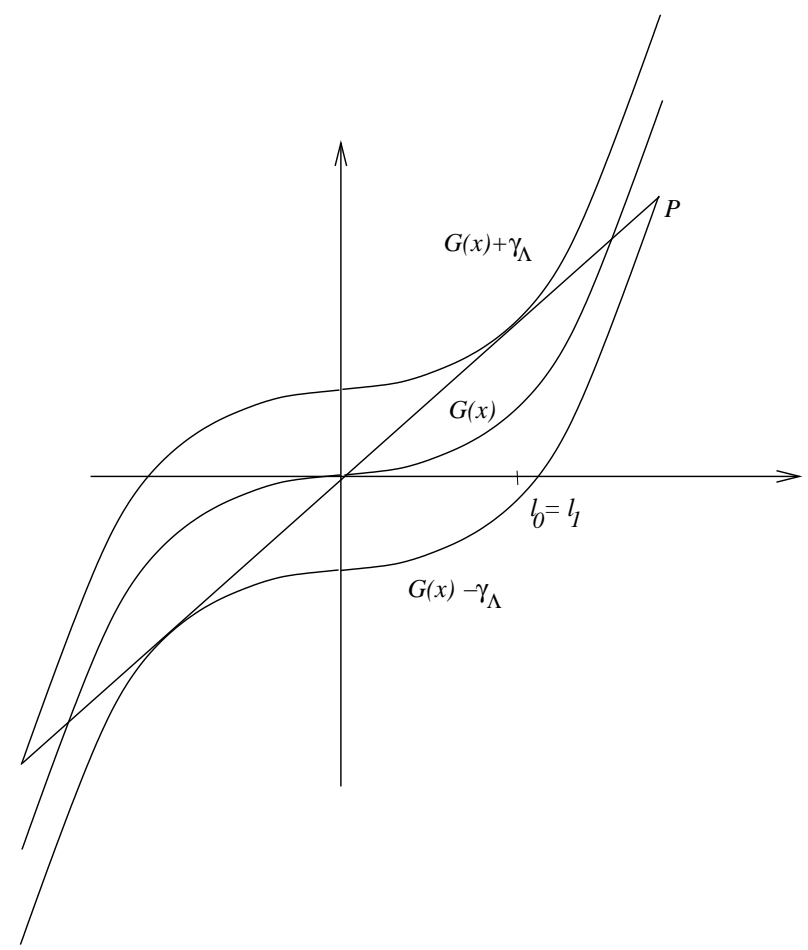

Fig. 1

\subsubsection{The tangency condition}

Here we introduce another notion important for our analysis. Let us suppose that $(\Gamma, \xi)$ is a solution and $I$ is a faceted region of $\Gamma$. We shall say that $(\Gamma, \xi)$ satisfies the tangency 
condition at $I$ if

$$
\left.\frac{\partial \xi}{\partial \tau}\right|_{\partial I}=0
$$

We notice here that $\frac{\partial \xi_{2}}{\partial \tau}=0$ always holds. What matters is the behavior of the first component of $\xi$.

In other words, the definition stipulates that if $\left(-L,-l_{1}\right),\left(-l_{0}, l_{0}\right),\left(l_{1}, L\right), l_{0} \leq l_{1}$ are inverse images of faceted regions and the tangency condition holds at each of them, then

$$
\frac{\partial}{\partial x} \xi_{1}\left( \pm l_{i}\right)=0, \quad i=0,1
$$

At this moment we have to make sure that we can evaluate $\frac{\partial \xi_{1}}{\partial x}$ at any point and if not, then we should explain the meaning of (2.19) and (2.20). Since we assumed (2.15) by Proposition 2.1 we deduce that $\xi$ is constant on $\left(-l_{1},-l_{0}\right)$ and $\left(l_{0}, l_{1}\right)$. In particular the following one-sided derivates exist $\frac{\partial \xi_{1}}{\partial x}{ }^{+}$at $-l_{1}, l_{0},{\frac{\partial \xi_{1}}{\partial x}}^{-}$at $-l_{0}, l_{1}$ and they are equal to zero.

Moreover, because the intervals $\left(-L, l_{1}\right),\left(-l_{0}, l_{0}\right),\left(l_{1}, L\right), l_{0} \leq l_{1}$ are inverse images of faceted regions it follows that $\frac{\partial \xi_{1}}{\partial x}$ is continuous there. As a result the remaining onesided derivatives at $\pm l_{i}, i=0,1$ are well-defined.

Later, we shall see that in the cases of interest (see e.g. Proposition 2.4) the derivative $\frac{\partial \xi_{1}}{\partial x}$ is everywhere well-defined and continuous.

The first application of the new notion is to make the following definition more handy. We shall say that $I$ a faceted region of $\Gamma$ has the maximality property, if $(\Gamma, \xi)$ satisfies the tangency condition at $I$.

Some comments are in order, one would expect that the word 'maximality' above refers to inclusion of sets. This is indeed so, but indirectly. If we define a function $G$ by formula

$$
G(x)=\int_{0}^{x} \sigma(s) d s
$$

and if we set $\eta(x)=G(x)-\xi_{1}(x)$, where $\xi$ is a solution to (2.11), then by the definition of $\mathcal{E}$ and Proposition $2.1 \eta$ is a minimizer of the functional $\int_{-L}^{L}\left|\frac{d}{d x} \eta(x)\right|^{2} d x$, with the constraint $|G(x)-\eta(x)| \leq \gamma\left(\mathbf{n}_{R}\right)$, see Fig. 1. We see that at the points, where (2.20) holds, the straight line emanating from point $P=\left(L, G(L)-\gamma\left(\mathbf{n}_{R}\right)\right)$ is tangent to the graph of $G$, hence the origin of the name of tangency conditions. Moreover, if we draw the line from $P$ to $\left(\lambda_{1}, G\left(\lambda_{1}\right)\right), \lambda_{1}>l_{1}=l_{0}$, (this is possible, after changing slightly $d$ ), then this line necessarily is not tangent to the graph of $G$ and the new facet is faster than the old one. Moreover, it is obvious from the picture that $\dot{R}_{1}>\dot{R}_{0}$, see Fig. 1 . This fact will be proven rigorously below.

Now, we have to show that these notions are useful. The proposition below serves this purpose.

Proposition 2.3 Let us assume that $\Gamma$ has exactly three disjoint faceted regions and $\left(-L, l_{1}\right)$, $\left(-l_{0}, l_{0}\right),\left(l_{1}, L\right), l_{0} \leq l_{1}$ are their inverse images. Moreover, $\xi$ is a solution to (2.11).

(a) If $\Gamma$ is a line segment, then the tangency condition holds on each faceted region.

(b) If $\Gamma$ is of the form (2.12) and $\lambda_{0}>l_{0}$ (respectively, $\lambda_{1}<l_{1}$ ), then the tangency condition holds at $\left(-l_{0}, l_{0}\right)$ (respectively, at $\left(-L,-l_{1}\right),\left(l_{1}, L\right)$ ). 
Proof. (a) In the present setting the minimization problem (2.11) is in fact an obstacle problem. Since $\xi_{1}(0)=0$ and $\xi_{1}(L)=\gamma\left(\mathbf{n}_{\Lambda}\right)$ we may directly apply [KS, Ch. II, Theorem $7.1]$ to conclude validity of (2.20).

(b) Since $\xi_{1}$ satisfies specific boundary conditions, we may apply the same argument as in (a) to deduce the same result.

Remark 2.1 The above mentioned result from [KS] may be explained by saying that a solution to an obstacle problem (2.11) meets the smooth obstacle tangentially.

Momentarily, we shall see further applications of these notions. The definition of $\mathcal{E}$, see formula (2.9), the setup of (2.11) as well as (2.16) suggest that $\xi$ is defined in terms of $\sigma$. Indeed, our next results assures us about it. At the same time we may write the final form of the evolution system we are going to study. In this process it is crucial for us that the tangency conditions hold at $l_{0}$ and $l_{1}$. Indeed, if we know this, then from (2.16) we deduce that $\dot{R}_{i}=\sigma\left(l_{i}\right)$ making Proposition 2.2 applicable.

Proposition 2.4 We assume that $\sigma$ satisfies (2.1) and (2.2) without explicit reference to the time dependence. Let us suppose that $(\Gamma, \xi)$ is a variational solution to (1.1), which at each time instant $t \geq 0 \Gamma(t)$ has exactly three faceted regions with the maximality property, their inverse images are $\left(-L,-l_{1}\right),\left(-l_{0}, l_{0}\right)$ and $\left(l_{1}, L\right)$. Furthermore, for all $t \geq$ 0 the assumptions of Proposition 2.1 (in particular (2.15)) hold and either of the following conditions are satisfied:

(i) $\Gamma$ is a graph of a constant function;

(ii) $l_{0}<\lambda_{0}$ and $\lambda_{1}<l_{1}$;

(iii) $l_{i}(t) \equiv l_{i 0}, i=0,1$.

Then,

(a) We have the following formula for $\xi_{1}$ for each time $t \geq 0$

$\xi_{1}(x)= \begin{cases}x\left(f_{0}^{x} \sigma(s) d s-f_{0}^{l_{0}} \sigma(s) d s\right)-\frac{x}{l_{0}} \gamma\left(\mathbf{n}_{\Lambda}\right) & \text { for } x \in\left[0, l_{0}\right) ; \\ -\gamma\left(\mathbf{n}_{\Lambda}\right) & \text { for } x \in\left[l_{0}, l_{1}\right] ; \\ (L-x)\left(f_{l_{1}}^{L} \sigma(s) d s-f_{x}^{L} \sigma(s) d s\right)+\gamma\left(\mathbf{n}_{\Lambda}\right)-2 \frac{L-x}{L-l_{1}} \gamma\left(\mathbf{n}_{\Lambda}\right) & \text { for } x \in\left(l_{1}, L\right],\end{cases}$

where we write $f_{A} f d \mu=\frac{1}{\mu(A)} \int_{A} f d \mu$. In addition, $\dot{R}_{0}, \dot{R}_{1}>0$.

(b) Equation (2.16) (and hence (1.1)) takes the following form,

$$
\begin{aligned}
& \dot{R}_{0}=f_{0}^{l_{0}} \sigma(s) d s+\frac{\gamma\left(\mathbf{n}_{\Lambda}\right)}{l_{0}} \quad \text { on }\left(-l_{0}, l_{0}\right) \\
& d_{t}=\sigma \quad \text { on } \quad\left[l_{0}, l_{1}\right] \\
& \dot{R}_{1}=\int_{l_{1}}^{L} \sigma(s) d s+\frac{2 \gamma\left(\mathbf{n}_{\Lambda}\right)}{L-l_{1}} \quad \text { on } \quad\left[-L,-l_{1}\right) \cup\left(l_{1}, L\right] .
\end{aligned}
$$

(c) The matching and the tangency conditions hold at each $t \geq 0$.

Proof. We start with (c), because it is used in the proof of (a). We notice that the matching condition is a result of continuity of $d(t, \cdot)$ in $[-L, L]$. We shall prove

$$
\frac{\partial}{\partial x} \xi_{1}\left(l_{i}\right)=0, \quad i=0,1
$$


Indeed, if $\Gamma$ is the graph of a constant function (resp. $l_{0}<\lambda_{0}$ and $\lambda_{1}<l_{1}$ ) this is the conclusion of Proposition 2.3 (a) (resp. Proposition 2.3 (b)). If $l_{i}(t)=$ const $_{i}, i=0,1$, then Proposition 2.2 and (2.16) imply that (2.23) holds.

(a) Due to the symmetry of the problem we consider only positive arguments $x \in[0, L]$. Let us integrate $\left(2.16_{1}\right)$ over $[0, x], x \leq l_{0}$, this yields

$$
\beta\left(\mathbf{n}_{\Lambda}\right) \dot{R}_{0} \cdot x=\int_{0}^{x} \sigma(s) d s-\xi_{1}(x),
$$

where we used $\xi_{1}(0)=0$. This formula for $x=l_{0}$ gives the value of $\dot{R}_{0}$, however at the moment we know only that $\xi\left(l_{0}\right)$ belongs to the boundary of $\partial \gamma\left(\mathbf{n}_{\Lambda}\right)$, i.e.

$$
\xi_{1}\left(l_{0}\right)= \pm \gamma\left(\mathbf{n}_{\Lambda}\right)
$$

We have to determine the sign in (2.25). Nonetheless, we may now eliminate $\dot{R}_{0}$ from (2.24). After doing so, we come to the following formula for $\xi_{1}(x), x \in\left[0, l_{0}\right]$.

$$
\xi_{1}(x)=x\left(f_{0}^{x} \sigma-f_{0}^{l_{0}} \sigma\right)+\frac{x}{l_{0}} \xi_{1}\left(l_{0}\right) .
$$

We recall that condition (2.7) implies that $\xi_{1}(L)=\gamma\left(\mathbf{n}_{\Lambda}\right)$, then a reasoning similar to that which lead us to (2.26) results in a formula for $\xi_{1}$ on $\left[l_{1}, L\right]$. It is

$$
\xi_{1}(x)=(L-x)\left(f_{l_{1}}^{L} \sigma(s) d s-f_{x}^{L} \sigma(s) d s\right)+\gamma\left(\mathbf{n}_{\Lambda}\right)+\frac{L-x}{L-l_{1}}\left(\xi_{1}\left(l_{1}\right)-\gamma\left(\mathbf{n}_{\Lambda}\right)\right) .
$$

Now, we since we assume that (2.15) holds we can use Proposition 2.1 to deduce that $\xi_{1}$ is constant over $\left[l_{0}, l_{1}\right]$, i.e. that $\xi_{1}\left(l_{1}\right)=\xi_{1}\left(l_{0}\right)$.

Let us calculate the derivative of $\xi_{1}$ :

$$
\begin{aligned}
& \frac{\partial \xi_{1}}{\partial x}(x)=\sigma(x)-f_{0}^{l_{0}} \sigma(s) d s+\frac{\xi_{1}\left(l_{0}\right)}{l_{0}} \quad \text { for } x \in\left[0, l_{0}\right] \\
& \frac{\partial \xi_{1}}{\partial x}(x)=\sigma(x)-f_{l_{1}}^{L} \sigma(s) d s+\frac{\gamma\left(\mathbf{n}_{\Lambda}\right)-\xi_{1}\left(l_{1}\right)}{L-l_{1}} \quad \text { for } x \in\left[l_{1}, L\right] .
\end{aligned}
$$

With this knowledge combined with the form of $\beta$ (see (1.5)) system (2.16) becomes

$$
f_{0}^{l_{0}} \sigma(s) d s-\frac{\xi_{1}\left(l_{0}\right)}{l_{0}}=\dot{R}_{0}, \quad \sigma=d_{t}, \quad f_{l_{1}}^{L} \sigma(s) d s+\frac{\gamma\left(\mathbf{n}_{\Lambda}\right)-\xi_{1}\left(l_{1}\right)}{L-l_{1}}=\dot{R}_{1}
$$

Since $\gamma\left(\mathbf{n}_{\Lambda}\right) \geq \xi_{1}\left(l_{1}\right)$ it follows from (2.29) that $\dot{R}_{1}>0$.

As we mentioned we have to determine the sign in (2.25). We notice that once we established the tangency condition (2.23), then (2.16) implies that $\sigma\left(l_{0}\right)=\dot{R}_{0}$. We notice that due to monotonicity of $\sigma$ the difference

$$
\sigma\left(l_{0}\right)-f_{0}^{l_{0}} \sigma(s) d s
$$

is always positive. Hence, (2.29) yields

$$
-\xi_{1}\left(l_{0}\right)=l_{0} \dot{R}_{0}-\int_{0}^{l_{0}} \sigma(s) d s>0,
$$


i.e. $\xi_{1}\left(l_{0}\right)=-\gamma\left(\mathbf{n}_{\Lambda}\right)$. Since $\xi$ is constant on $\left[l_{0}, l_{1}\right]$ we conclude that $\xi_{1}\left(l_{1}\right)=-\gamma\left(\mathbf{n}_{\Lambda}\right)$ as well. Only now we may infer that (2.29) implies that $\dot{R}_{0}>0$.

The assumed symmetry of the problem implies that for $x<0$ we have

$$
\xi_{1}(x)=-\xi_{1}(-x),
$$

hence in particular, $\xi_{1}\left(-l_{1}\right)=-\xi_{1}\left(l_{1}\right)=\gamma\left(\mathbf{n}_{\Lambda}\right)$.

(b) If we insert $\xi_{1}\left(l_{0}\right)=\xi_{1}\left(l_{1}\right)=-\gamma\left(\mathbf{n}_{\Lambda}\right)$ into (2.29) we infer (2.22).

Let us stress that we have not used that the full power of the assumption that in the solution $(\Gamma, \xi)$ the Cahn-Hoffman vector is a solution to (2.11). We will do this now. This will be one of the key observations.

Corollary 2.1 Let us suppose that $(\Gamma, \xi)$ is a variational solution to (1.1) and the assumptions of Proposition (2.1) are satisfied. Then following three conditions are equivalent:

(a) the tangency conditions, i.e. $\frac{\partial \xi}{\partial x}\left(l_{i}\right)=0, i=0,1$;

(b) $\dot{R}_{i}=\sigma\left(t, l_{i}\right), i=0,1$;

(c)

$\sigma\left(l_{0}\right)=f_{0}^{l_{0}} \sigma(s) d s+\frac{\gamma\left(\mathbf{n}_{\Lambda}\right)}{l_{0}}, \quad \sigma\left(l_{1}\right)=f_{l_{1}}^{L} \sigma(s) d s-\frac{2 \gamma\left(\mathbf{n}_{\Lambda}\right)}{L-l_{1}} \quad$ and $\quad \xi_{1}\left(l_{i}\right)=-\gamma\left(\mathbf{n}_{\Lambda}\right)$.

Proof. We first show equivalence of (a) and (b). Indeed, equations $(2.16)_{i}, i=1,2$ imply that (a) holds iff (b) is satisfied.

Now, it is more convenient to show equivalence of (a) and (c). In fact the proof of Proposition 2.4 yields $(\mathrm{a}) \Rightarrow(\mathrm{c})$, see (2.28). In order to show the converse we note that (2.28) was derived from (2.16) without making any use of the additional assumptions on $\xi$. If we know (c), then (2.28) immediately implies (a).

Remark 2.2. We stress that system (2.16) was derived for variational solutions.

Let us now draw a conclusion, which permits us to compare $\dot{R}_{0}$ and $\dot{R}_{1}$.

Corollary 2.2 If $(\Gamma, \xi)$ is a variational solution to (1.1) and the assumptions of Proposition 2.4 are satisfied, then $\dot{R}_{0} \leq \dot{R}_{1}$.

Proof. By assumption the function $d(t, \cdot)$ is continuous in $[-L, L]$. It follows from (2.22) that

$$
\dot{R}_{0}=f_{0}^{l_{0}} \sigma(s) d s+\frac{\gamma\left(\mathbf{n}_{\Lambda}\right)}{l_{0}}, \quad d_{t}\left(t, l_{0}\right)=\sigma\left(l_{0}\right) .
$$

Due to the tangency condition $\left(2.30_{1}\right)$ we conclude that

$$
\dot{R}_{0}=d_{t}\left(t, l_{0}\right) .
$$

Monotonicity of $\sigma$ and the other tangency condition $\left(2.30_{2}\right)$ imply that

$$
\dot{R}_{0}=\sigma\left(l_{0}\right) \leq \sigma\left(l_{1}\right)=\dot{R}_{1} .
$$

Hence the claim. 


\subsection{Analysis of ODE (2.22)}

The forthcoming analysis constitutes the core of the paper. In order to be able to solve (2.22) we have to make sure it is a closed system. This is indeed so as long as functions $l_{0}(t), l_{1}(t)$ are given with $l_{0}(0)=l_{00}, l_{1}(0)=l_{10}$. Once we guarantee this we can try to solve (2.22). Let us stress however, that in order to construct solutions to (1.1) we must find $\xi$ and prove that it is a solution to (2.11).

We shall start with the first problem of determining $l_{0}$ and $l_{1}$. However, it is advantageous to consider a slightly more general situation and draw interesting conclusions afterward.

Proposition 2.5 Let us suppose that $\sigma=\sigma(x, \mu)$ and $\sigma, \frac{\partial \sigma}{\partial x} \in C(\mathbb{R} \times U)$, where $U \subset \mathbb{R}^{n}$ is open. We assume that $\sigma(-x, \mu)=\sigma(x, \mu)$ and $\frac{\partial \sigma}{\partial x}(x, \mu)>0$ for all $x>0, \mu \in U$, and $0<l_{00} \leq l_{10}<L$.

If the tangency condition $\left(2.30_{i+1}\right), i=0,1$ is satisfied for $\left(l_{i 0}, \mu_{0}\right) \in(-L, L) \times U$, then there exists a neighborhood $U_{0} \subset U$ of $\mu_{0}$ and continuous functions $U_{0} \ni \mu \rightarrow$ $l_{0}(\mu), l_{1}(\mu)$, such that (2.30) hold for all $\mu \in U$ and $l_{i}(\mu), i=0,1$. Moreover, if $l_{00}<l_{10}$, then $l_{0}(\mu)<l_{1}(\mu)$ for all $\mu \in U_{0}$. If in addition $\sigma \in C^{1}(\mathbb{R} \times U)$, then $l_{i}, i=0,1$ are of class $C^{1}$.

Proof. We first consider $l_{0}$. In fact it is sufficient to check the assumptions of the Implicit Function Theorem (see e.g. [N, Theorem 2.7.2]) to conclude our claim. Let us set $F\left(l_{0}, \mu\right)=\int_{0}^{l_{0}} \sigma(s, \mu) d s+\gamma\left(\mathbf{n}_{\Lambda}\right)-l_{0} \sigma\left(l_{0}, \mu\right)$. Then $F\left(l_{00}, \mu_{0}\right)=0$. We calculate $\frac{\partial F}{\partial l_{0}}$ :

$$
\left.\frac{\partial F}{\partial l_{0}}\left(l_{0}, \mu\right)\right|_{\left(l_{00}, \mu_{0}\right)}=\sigma\left(l_{00}, \mu_{0}\right)-l_{00} \frac{\partial \sigma}{\partial l_{0}}\left(l_{00}, \mu_{0}\right)-\sigma\left(l_{00}, \mu_{0}\right)=-l_{00} \frac{\partial \sigma}{\partial l_{0}}\left(l_{00}, \mu_{0}\right) \neq 0 .
$$

The Implicit Function Theorem implies also differentiability of $l_{i}$ 's with respect to $\mu_{k}$ if $F$ is of class $C^{1}$ with respect to $\mu_{k}$. It follows that

$$
\frac{\partial l_{0}}{\partial \mu_{j}}=\left(l_{0} \frac{\partial \sigma}{\partial x}\right)^{-1}\left(\int_{0}^{l_{0}} \frac{\partial \sigma}{\partial \mu_{j}}(s, \mu) d s-l_{0} \frac{\partial \sigma}{\partial \mu_{j}}\right) \quad j=1, \ldots, n .
$$

The case of $l_{1}$ is similar to the previous one. We consider it in detail, because we want to establish differentiability of $l_{1}$ with respect to $L$, i.e. the endpoint of the interval of definition of $d$. Namely, we set

$$
F\left(l_{1}, L, \mu\right)=\int_{l_{1}}^{L} \sigma(s, \mu) d s-2 \gamma\left(\mathbf{n}_{\Lambda}\right)-\left(L-l_{1}\right) \sigma\left(l_{1}, \mu\right) .
$$

We easily check that

$$
\left.\frac{\partial F}{\partial L}\right|_{\left(l_{10}, \mu_{0}, L_{1}\right)}=\sigma\left(L_{1}, \mu_{0}\right)-\sigma\left(l_{10}, \mu_{0}\right)>0 .
$$

Hence, $\frac{\partial l_{1}}{\partial L}$ exists and it is continuous.

If $l_{00}=l_{10}$, then we cannot claim anymore that $l_{0}(\mu) \leq l_{1}(\mu)$ for $\mu \neq \mu_{0}$. However, due to (2.31) the quantities $l_{0}(\mu)$ and $l_{1}(\mu)$ remain well-defined. We will depend upon it later. 
It is important to notice that due to the above proposition the tangency condition propagates. This gives us a chance to solve the problem, if $l_{0}(t)$ and $l_{1}(t)$ are non-trivial functions of time (see next section).

In the particular case considered in the present section $l_{0}(t)$ and $l_{1}(t)$ are constants. Indeed if $U=\mathbb{R}_{+}, \mu=t$, and $\frac{\partial \sigma}{\partial t} \equiv 0$, then (2.32) implies that

$$
\frac{d}{d t} l_{0}(t) \equiv 0 \quad \text { and } \quad \frac{d}{d t} l_{1}(t) \equiv 0 .
$$

Let us stress that our insisting on the tangency condition at $t=0$ makes the initial data quite special, but in complete accordance with our previous results, see [GR3], [GR4]. It is another question what happens if the data violate the tangency condition at $t=0$. We will make comments on this in subsection 2.4 below. We are rather inclined to think that such situation does not occur if (1.1) is coupled to a diffusion field and this case arises in [GR3], [GR4]. This topic requires further investigations.

We are now able to state the main existence result of this section yielding existence of solution to (2.22) as well as (1.1). Uniqueness will be shown in a separate theorem.

Theorem 2.1 Let us assume (1.5) and consider system (2.22) augmented with initial condition $\left(\Gamma_{0}, \xi_{0}\right)$, where $\Gamma_{0}=\tilde{d}_{0}$, (see (2.14)), $d_{0}$ is Lipschitz continuous of the form (2.12) i.e. the real numbers $l_{00}, l_{10}, R_{00}, R_{10}$ are given. In addition the exceptional set $E_{Z}$ defined in Proposition 2.1 has $\mathcal{H}^{1}$ measure zero at time $t=0$. Moreover $\xi_{0} \in \mathcal{D}$ is a solution to (2.11). We assume that $\sigma \in C^{1}([-L, L]), \sigma(-x)=\sigma(x)$ and $\frac{d \sigma}{d x}>0$ for $x>0$. Moreover, we impose the following conditions:

(a) $l_{00} \leq l_{10}, \quad 0<R_{00} \leq R_{10}$ and $d_{0}(\cdot)$ is increasing for $x \geq 0$ and $d_{0} \in$ $C^{1}\left(\left[l_{0}, l_{1}\right]\right)$.

(b) there are exactly three faceted regions of $\Gamma(0)$, namely $\left(-L,-l_{10}\right),\left(-l_{00}, l_{00}\right)$ and $\left(l_{10}, L\right)$ are their inverse images;

(c) the matching conditions (2.17) hold at $t=0$, i.e. $R_{00}=d_{0}\left(l_{00}\right)$ and $R_{10}=d_{0}\left(l_{10}\right)$;

(d) the tangency conditions (2.30) are satisfied at $t=0$, i.e.

$$
\left.\sigma\left(l_{00}\right)=f_{0}^{l_{00}} \sigma(s) d s+\frac{\gamma\left(\mathbf{n}_{\Lambda}\right)}{l_{00}}, \quad \sigma\left(l_{10}\right)=f_{l_{10}}^{L} \sigma(s)\right) d s-\frac{2 \gamma\left(\mathbf{n}_{\Lambda}\right)}{L-l_{1}} .
$$

Then,

(i) There exists a local in time solution to (2.22), $R_{0}, R_{1}$ and $d(t, \cdot) \in C^{1}\left(\left[l_{0}, l_{1}\right]\right)$ (and $\left.d(t, \cdot) \in C^{1}\left(\left[-l_{1},-l_{0}\right]\right)\right)$ and $d(t, \cdot)$ is strictly increasing, whose derivative $d_{x}(t, x)$ never vanishes for $x \in\left(-l_{1},-l_{0}\right) \cup\left(l_{0}, l_{1}\right)$, i.e. the exceptional set $E_{Z}$ is a subset (possibly empty) of $\left\{ \pm l_{0}, \pm l_{1}\right\}$ for $t>0$;

(ii) The matching (2.17) and tangency (2.30) conditions hold for all times $t>0$, that is if we extend $d(t, \cdot)$ to $[-L, L]$ by

$$
\bar{d}(t, x)= \begin{cases}R_{1}(t) & \text { if }|x| \in\left(l_{1}, L\right) \\ d(t, x) & \text { if }|x| \in\left[l_{0}, l_{1}\right] \\ R_{0}(t) & \text { if }|x| \in\left[0, l_{0}\right)\end{cases}
$$

then $\bar{d}(t, \cdot)$ is Lipschitz continuous on $[-L, L]$. (Subsequently we drop the bar over the extension.) 
Proof. (i) We begin with noticing that Proposition 2.5 guarantees existence of uniquely defined functions $l_{0}(t)$ and $l_{1}(t)$. Independence $\sigma$ from $t$ yields that

$$
l_{0}(t) \equiv l_{00} \quad \text { and } \quad l_{1}(t) \equiv l_{10} .
$$

Once we are provided with $l_{0}$ and $l_{1}$ we can solve (2.22). Existence of global in time solutions to this system follows trivially from the fact that the right-hand-side of (2.22) is independent from $R_{0}, R_{1}$ and $d$. However, the condition $\left|d_{x}\right| \leq 1$ makes the solution restricted to a time interval $[0, T)$.

(ii) The continuous dependence of solutions upon $x \in\left(-l_{1},-l_{0}\right) \cup\left(l_{0}, l_{1}\right)$ follows from continuity of the RHS of (2.22).

We will check that the matching condition (2.17) holds for $t>0$. Since $R_{i 0}-d_{0}\left(l_{i 0}\right)=$ $0, i=0,1$ it is sufficient to check that the derivative of $R_{i}(t)-d\left(t, l_{i}(t)\right)$ vanishes. We have for $i=0,1$,

$$
\frac{d}{d t}\left(R_{i}(t)-d\left(t, l_{i}(t)\right)\right)=\dot{R}_{i}-\frac{\partial d}{\partial t}\left(t, l_{i 0}\right)=\dot{R}_{i}-\sigma\left(l_{i 0}\right) .
$$

Now, we notice the tangency conditions holds for $t \geq 0$. This follows trivially from (2.30) and the fact that $\sigma$ and $l_{0}, l_{1}$ are independent of time. Hence, the right-hand-side vanishes. This implies that the extended $d$ is indeed Lipschitz continuous on $[-L, L]$. This follows from the smooth dependence of solutions of (2.22) on parameters.

(iii) We have to investigate $\xi$ given by (2.21). Our claim will follow once we establish the Lemma below.

Finally, condition (b) in the definition of solution to (1.1) is satisfied because $\xi$ is independent of time and by formulas (2.26), (2.27) it belongs to $\mathcal{D}$.

Lemma 2.1 If $\xi$ is defined by (2.21) and the tangency conditions hold, then this $\xi$ is a solution to (2.11). In addition, the exceptional set $E_{Z}$ contains at most four points.

Proof. We shall compare $\mathcal{E}(\xi+h)$ and $\mathcal{E}(\xi)$. We have to examine the assumption that $\xi+h$ is in $\mathcal{D}$. Let us write $h(\tilde{d}(t, x))=\left(h_{1}(x), h_{2}(x)\right)$. First, since $\partial \gamma\left(\mathbf{n}_{\Lambda}\right) \cap \partial \gamma\left(\mathbf{n}_{R}\right)=\{p\}$, then $\left.\left.h_{1}( \pm L)\right)=0=h_{2}( \pm L)\right)$. Secondly, the structure of $\mathcal{D}$ implies that $h_{2}(x)=0$ for all $x \in[-L, L]$. Moreover, due to $\xi_{1}(x)=-\gamma\left(\mathbf{n}_{\Lambda}\right)$ at $x=l_{i}, i=0,1$ we have $h_{1}\left(l_{i}\right) \geq 0$. By a similar argument $h_{1}\left(-l_{i}\right) \leq 0$.

In general $\mathcal{E}(\xi)$ is a curvilinear integral, which can be written as

$$
\mathcal{E}(\xi)=\int_{-L}^{L} \frac{1}{2}\left|\sigma(x)-\tau \cdot \frac{\partial \xi}{\partial \tau}(x)\right|^{2} \sqrt{1+d_{x}^{2}} d x .
$$

On inverse images of faceted regions this integral simplifies because $\tau \cdot \frac{\partial \xi}{\partial \tau}=\frac{\partial \xi_{1}}{\partial x}$ and $d_{x}=0$.

On $\left(l_{0}, l_{1}\right)$ the formula for solutions $d$

$$
d(t, x)=d_{0}(x)+t \sigma(x)
$$

is valid, hence $d$ is strictly increasing and due to $\sigma^{\prime}(x)>0$ for $x>0$ the derivative $\frac{\partial d}{\partial x}(t, x)$ never vanishes on $\left(l_{0}, l_{1}\right)$. As a result, for each $t>0$ the exceptional set $E_{Z}$ contains at most four point, hence Proposition 2.1 yields $\operatorname{div}_{S} \xi=0$ on $\left(-l_{1},-l_{0}\right) \cup\left(l_{0}, l_{1}\right)$. 
In addition we deduce that $\mathbf{n} \neq \mathbf{n}_{\Lambda}, \mathbf{n}_{R}$ there, hence $\partial \gamma(\mathbf{n})$ is a singleton, thus $h=0$ on $\left(-l_{1},-l_{0}\right) \cup\left(l_{0}, l_{1}\right)$.

For our calculations we have to know the behavior of the $\operatorname{difference} \sigma-\operatorname{div}_{S} \xi$ on $\left[-L,-l_{1}\right],\left[-l_{1},-l_{0}\right],\left[-l_{0}, l_{0}\right],\left[l_{0}, l_{1}\right],\left[l_{1}, L\right]$. Due to symmetries involved we may consider only positive arguments. First we take $x \in\left[0, l_{0}\right]$, thus $\operatorname{div}_{S} \xi=\frac{\partial \xi_{1}}{\partial x}$. Once we use formula (2.21) we can immediately see

$$
\sigma-\frac{\partial \xi_{1}}{\partial x}=f_{0}^{l_{0}} \sigma(s) d s+\gamma\left(\mathbf{n}_{\Lambda}\right)=\sigma\left(l_{0}\right)
$$

holds on $\left[0, l_{0}\right]$, hence on $\left[-l_{0}, l_{0}\right]$. The last equality in $(2.35)$ is a consequence of the tangency condition.

Next, we consider $\left[-l_{1},-l_{0}\right] \cup\left[l_{0}, l_{1}\right]$. Here we obviously have

On interval $\left[l_{1}, L\right]$ it holds

$$
\sigma(x)-\frac{\partial \xi_{1}}{\partial x} \equiv \sigma(x)
$$

$$
\sigma-\frac{\partial \xi_{1}}{\partial x}=f_{l_{1}}^{L} \sigma(s) d s-\frac{2 \gamma\left(\mathbf{n}_{\Lambda}\right)}{L-l_{1}}=\sigma\left(l_{1}\right),
$$

where we again used the tangency condition. The same result is valid on $\left[-L, l_{1}\right]$.

After collecting the above information we can see that,

$$
\begin{aligned}
\mathcal{E}(\xi+h)= & \frac{1}{2}\left(\int_{-l_{0}}^{l_{0}}\left|\sigma\left(l_{0}\right)-\frac{\partial h_{1}}{\partial x}\right|^{2} d x+\left(\int_{-l_{1}}^{-l_{0}}+\int_{l_{0}}^{l_{1}}\right)|\sigma(x)|^{2} \sqrt{1+d_{x}^{2}} d x\right) \\
& +\frac{1}{2}\left(\int_{-L}^{-l_{1}}+\int_{l_{1}}^{L}\right)\left|\sigma\left(l_{1}\right)-\frac{\partial h_{1}}{\partial x}\right|^{2} d x .
\end{aligned}
$$

Hence,

$$
\begin{aligned}
& \mathcal{E}(\xi+h)-\mathcal{E}(\xi)= \\
& \quad \int_{-l_{0}}^{l_{0}}\left[\frac{1}{2}\left(\frac{\partial h_{1}}{\partial x}\right)^{2}-\sigma\left(l_{0}\right) \frac{\partial h_{1}}{\partial x}\right] d x+\left(\int_{-L}^{-l_{1}}+\int_{l_{1}}^{L}\right)\left[\frac{1}{2}\left(\frac{\partial h_{1}}{\partial x}\right)^{2}-\sigma\left(l_{1}\right) \frac{\partial h_{1}}{\partial x}\right] d x .
\end{aligned}
$$

Integration yields

$$
\mathcal{E}(\xi+h)-\mathcal{E}(\xi) \geq-\left.\sigma\left(l_{0}\right) h\right|_{-l_{0}} ^{l_{0}}-\sigma\left(l_{1}\right)\left(\left.h\right|_{l_{1}} ^{L}+\left.h\right|_{-L} ^{-l_{1}}\right) .
$$

The last conclusion follows from $\sigma^{\prime}(x)>0$ for $x>0, h\left(l_{i}\right) \geq 0$ and $\sigma^{\prime}(x)<0$ for $x<0, h\left(-l_{i}\right) \leq 0$.

\section{Remarks 2.3.}

(1) We admit $R_{00}=R_{10} \equiv d_{0}$.

(2) We may also admit $l_{00}=l_{10}$, i.e. the line segment $\Gamma$ contains three distinct faceted regions. It is possible to handle this case, because $l_{0}, l_{1}$ are constant, hence the facet $\Gamma$, which is initially flat due to the matching conditions

$$
R_{0}(0)=R_{00}=d_{0}\left(l_{00}\right)=d_{0}\left(l_{10}\right)=R_{10}=R_{1}(0)
$$

will stay flat without bending non bending.

(3) Our arguments used the fact that $\sigma_{x}$ has a sign, thus a similar conclusions may be drawn if $\sigma_{x}(x)<0$ for $x>0$ after making some changes like reversing the inequality signs.

(4) In subsection 2.4 below we study the data violating the tangency condition at $t=0$. 


\subsection{Uniqueness of solutions}

At last, we will show uniqueness of solutions, the proof is valid for a slightly broader class of solutions than we considered. If $(d, \xi)$ is a solution to (1.1), then for all $t \geq 0$ we can write

$$
(-L, L)=Z(t) \cup N(t) \cup D(t)
$$

where

$$
\begin{gathered}
Z(t)=\left\{x \in(-L, L): d_{x}(t, x)=0\right\}, \quad N(t)=\left\{x \in(-L, L): d_{x}(t, x) \neq 0\right\}, \\
D(t)=\{x \in(-L, L): d(t, \cdot) \text { is not differentiable at } x\} .
\end{gathered}
$$

The restriction will be stated in term of the set $N(t)$.

Theorem 2.2 Let us assume that $\beta$ satisfies (1.5), $\left(\Gamma^{i}, \xi^{i}\right), i=1,2$ are two solutions to (1.1) (not necessarily variational) defined on $[0, T)$ and $d^{1}(0, \cdot)=d^{2}(0, \cdot), \xi^{1}(0, \cdot)=$ $\xi^{2}(0, \cdot)$. If in addition

$$
\mathcal{H}^{1}(N(t) \backslash \operatorname{int} N(t))=0,
$$

then, $d^{1}(t, \cdot)=d^{2}(t, \cdot), \xi^{1}(t, \cdot)=\xi^{2}(t, \cdot)$ for all $t \in[0, T)$.

Remark 2.4. The assumption (2.37) is obviously satisfied by solutions yielded by Theorem 2.1.

Proof. We recall that $\xi_{2}$ must be constant. We notice that by (2.37) $\xi$ assumes a fixed value on each connected component of int $N(t)$ and its closure. Hence,

$$
0=\tau \cdot \frac{\partial \xi}{\partial \tau}=\frac{\partial \xi_{1}}{\partial x}
$$

Moreover, on the set $\left\{d_{x}=0\right\}$ we have $\tau=\mathbf{e}_{1}$. If we recall (2.37) and $\mathcal{H}^{1}(D(t))=0$, then we conclude

$$
\tau \cdot \frac{\partial \xi}{\partial \tau}=\frac{\partial \xi_{1}}{\partial x}
$$

in $(-L, L)$ in the distribution sense. This is why we may say that $\left(\Gamma^{i}, \xi^{i}\right), i=1,2$ satisfy the equation

$$
\frac{\partial d^{i}}{\partial t}(t, x)=\sigma(x)-\frac{\partial \xi_{1}^{i}}{\partial x}, \quad i=1,2 .
$$

Taking the difference of these two equations, multiplying by $d^{2}-d^{1}$, after integration over $[-L, L]$ we obtain

$$
\frac{1}{2} \frac{d}{d t} \int_{-L}^{L}\left(d^{2}-d^{1}\right)^{2}(x) d x=\int_{-L}^{L}\left(-\frac{\partial \xi_{1}^{2}}{\partial x}+\frac{\partial \xi_{1}^{1}}{\partial x}\right)\left(d^{2}-d^{1}\right)(x) d x
$$

The integration by parts and the boundary conditions (2.7) on $\xi^{i}$ at $x= \pm L$ lead us to

$$
\frac{1}{2} \frac{d}{d t}\left\|d^{2}-d^{1}\right\|_{L^{2}}^{2}=\int_{-L}^{L}\left(\xi_{1}^{2}-\xi_{1}^{1}\right)\left(d_{x}^{2}-d_{x}^{1}\right)(x) d x .
$$

Now, we notice that the integrand equals to the following inner product

$$
-\left(\xi^{2}-\xi^{1}\right) \cdot\left(d_{x}^{2}-d_{x}^{1}, 0\right)=: I .
$$


Since $\xi^{i}(x) \in \partial \gamma\left(\left(-d_{x}^{i}, 1\right)\right)$, because $\mathbf{n}(x)=\left(-d_{x}^{i}, 1\right) / \sqrt{1+\left(d_{x}^{i}\right)^{2}}$, we conclude by monotonicity of the subdifferential that $I \leq 0$. Hence

$$
\frac{1}{2} \frac{d}{d t}\left\|d^{2}-d^{1}\right\|_{L^{2}}^{2} \leq 0
$$

subsequently $d^{1}(t, \cdot)=d^{2}(t, \cdot)$ for $t>0$.

We shall show that $\xi^{1}$ and $\xi^{2}$ coincide. For this purpose we integrate (2.38) over $\left[t_{1}, t_{2}\right]$,

$$
d^{i}\left(t_{2}, x\right)-d^{i}\left(t_{1}, x\right)=\left(t_{2}-t_{1}\right) \sigma(x)-\int_{t_{1}}^{t_{2}} \frac{\partial \xi_{1}^{i}}{\partial x}(s, x) d s,
$$

for a.e. $x \in[-L, L]$. Since the left-hand-sides are equal for $i=1,2$ we conclude that

$$
\frac{\partial \xi_{1}^{1}}{\partial x}=\frac{\partial \xi_{1}^{2}}{\partial x}
$$

and finally $\xi^{1}=\xi^{2}$, as desired.

Remark 2.5. The argument depends upon the coincidence $\tau \cdot \frac{\partial \xi}{\partial \tau}=\frac{\partial \xi_{1}}{\partial x}$ and $\xi(x) \in \partial \gamma(\mathbf{n})$, in particular no information on the structure of $\xi$ is needed.

We could adopt a general view point of the semigroup theory. After defining a functional $\Phi$ on $L^{2}(-L, L)$ by the formula

$$
\Phi(d)= \begin{cases}\int_{-L}^{L}\left(\gamma\left(\left(-d_{x}, 1\right)\right)+\sigma d\right) d x & \text { if } d \in B V(-L, L) \\ +\infty & \text { otherwise. }\end{cases}
$$

we may write equation (1.1) as a general gradient flow

$$
\dot{d}+\partial \Phi(d) \ni 0 .
$$

This is so, because $\Phi$ is proper, convex and lower semicontinuous. The last is obvious once we realize that $\Phi$ is basically the semi-norm of $d$ in $B V$, see e.g. [Z].

The key point in our definition of solution is that at each $t \geq 0, \xi(t, \cdot)$ is a solution to a variational principle (2.11). It is rather easy to check that solutions to (2.11) yield canonical section to $\partial \Phi$. It is also well-known that the right derivative $d_{t}^{+}$in (2.39) equals to the canonical section (see $[\mathrm{Br}],[\mathrm{Ba}]$ for more details). In other words, our solutions is the semigroup solution hence it is unique. We will not elaborate upon it because we will not use it.

\subsection{Data violating the tangency condition}

We now consider the initial data for (2.22) which do not satisfy the tangency conditions. By Corollary 2.1 we know that for a variational solution the tangency conditions are equivalent to

$\sigma\left(l_{0}\right)=f_{0}^{l_{0}} \sigma(s) d s+\frac{\gamma\left(\mathbf{n}_{\Lambda}\right)}{l_{0}}, \quad \sigma\left(l_{1}\right)=f_{l_{1}}^{L} \sigma(s) d s-\frac{2 \gamma\left(\mathbf{n}_{\Lambda}\right)}{L-l_{1}} \quad$ and $\xi\left(l_{i}\right)=-\gamma\left(\mathbf{n}_{\Lambda}\right)$.

Since the discussion of all inverse images of faceted regions, i.e. $\left(-l_{0}, l_{0}\right),\left(-L,-l_{1}\right)$ and $\left(l_{1}, L\right)$ is analogous we restrict our attention only to the first one, while leaving the details in the second case to the reader. 
No matter what is the $t$ dependence of $\sigma$ we expect that if any of the conditions $(2.40)_{1,2}$ is violated, then the corresponding $l_{i}$ will depend upon time in a non-trivial way. Thus, in order to close system (2.22) we have to find an equation for $l_{0}$. Our starting point is the observation that the matching condition must hold, i.e. $R_{0}(t)=d\left(t, l_{0}(t)\right)$ for all $t \geq 0$. Assuming that $d(t, \cdot)$ has the right derivative $d_{x}^{+}$at $x=l_{0}(t)$ we find that

$$
\begin{aligned}
0 & \left.=d_{t}\left(t, l_{0}(t)\right)+d_{x}^{+}\left(t, l_{0}(t)\right) \dot{l}_{0}(t)\right)-\dot{R}_{0}(t) \\
& =\sigma\left(t, l_{0}(t)\right)-\dot{R}_{0}(t)+d_{x}^{+}\left(t, l_{0}(t)\right) \dot{l}_{0}(t)
\end{aligned}
$$

Apparently, we have two cases of violation the tangency conditions:

$$
\text { (i) } \sigma\left(t, l_{0}(t)\right)-\dot{R}_{0}(t)<0
$$

and

(ii) $\sigma\left(t, l_{0}(t)\right)-\dot{R}_{0}(t)>0$.

In the first case the facet is faster than the curved part, hence the facet grows at its expense. If we knew that $d_{x}^{+}\left(t, l_{0}(t)\right)>0$, then we could find $i_{0}(t)$,

$$
\dot{l}_{0}(t)=\left(\dot{R}_{0}(t)-\sigma\left(t, l_{0}(t)\right)\right) / d_{x}^{+}\left(t, l_{0}(t)\right)
$$

and indeed $i_{0}>0$. Equation (2.41) will be well-posed after we learn how to express $d_{x}^{+}\left(t, l_{0}(t)\right)$ in term of data. For this purpose we recall the formula

$$
d\left(t, l_{0}(t)\right)=d\left(0, l_{0}(t)\right)+\int_{0}^{t} \sigma\left(s, l_{0}(t)\right) d s .
$$

Hence,

$$
\left.d_{x}^{+}(t, x)\right|_{x=l_{0}(t)}=\left.\left(d_{0}\right)_{x}^{+}(x)\right|_{x=l_{0}(t)}+\left.\int_{0}^{t} \frac{\partial \sigma}{\partial x}(s, x)\right|_{x=l_{0}(t)} d s .
$$

Because of our assumptions on $\sigma$ we conclude that $d_{x}^{+}\left(t, l_{0}(t)\right)>0$. After recalling the formula for $\dot{R}_{0}$ we arrive at the final equation for $l_{0}(t)$, assuming that $\sigma$ is independent of time,

$$
i_{0}=\left(f_{0}^{l_{0}} \sigma(s) d s+\frac{\gamma\left(\mathbf{n}_{\Lambda}\right)}{l_{0}}-\sigma\left(l_{0}\right)\right) /\left(d_{x}^{+}\left(0, l_{0}\right)+t \frac{\partial \sigma}{\partial x}\left(l_{0}(t)\right)\right) .
$$

As long as we do not assume higher regularity $\left(\right.$ e.g. $\left.C^{1,1}\right)$ of $d_{0}(\cdot)$ and of $\sigma$ we are not able to conclude uniqueness of solutions to (2.43).

We are ready to state the existence result for case (i) above, it is formulated for the inner inverse image of faceted region $\left(-l_{0}, l_{0}\right)$, the other one is handled in a similar way. For simplicity we assume that the other faceted region satisfies the tangency condition (2.40).

Theorem 2.3 Let us suppose that all the assumptions of Theorem 2.1 except the tangency condition $(2.40)_{1}$, but instead the following inequality is satisfied

$$
\sigma\left(t, l_{0}(t)\right)-f_{0}^{l_{0}} \sigma(s) d s+\frac{\gamma\left(\mathbf{n}_{\Lambda}\right)}{l_{0}}<0 .
$$

Moreover, we assume that $d_{0} \in C^{1,1}\left(\left[l_{00}, l_{10}\right]\right)$ and $\sigma \in C^{1,1}$. Then there is a local in time variational solution to (1.1) and it is unique, however at no time $t>0$ condition (2.40) 1 holds. 
Proof. System (2.22) coupled to (2.43) forms a new closed system of ODE's augmented with initial data. Because we assumed that $\frac{\partial d_{0}}{\partial x}$ is Lipschitz continuous, then this system uniquely is solvable. Moreover, the solution depends on parameter $x$ in a differentiable manner.

We have to check that the matching condition holds. It is sufficient to show that

$$
\frac{d}{d t}\left(R_{0}(t)-d\left(t, l_{0}(t)\right)\right)=0,
$$

for $t \geq 0$. Indeed, by equation (2.41) and (2.43) identity (2.44) holds as desired.

The tangency condition cannot hold for any $t>0$. If it did at $t=t_{c r}$, then by (2.33) we would have $l_{0}(t) \equiv l_{0}\left(t_{c r}\right)$. Since we can solve ODE's backward in time, then by Theorem 2.1 we would conclude that the tangency conditions holds at $t=0$ which is a contradiction.

In order to show that we have constructed a variational solution we have to show that $\xi$ is a minimizer of (2.11). The argument presented in the proof of Lemma 2.1 depends upon the tangency condition, now violated, hence it must be adjusted. In order to simplify the exposition we assume that the tangency conditions hold on $\left(l_{1}, L\right)$. Let us set

$$
\delta=\frac{1}{2}\left(\dot{R}_{0}-\sigma\left(l_{0}\right)\right)>0
$$

and consider $h \geq$ such that

$$
\left\|\frac{\partial h}{\partial x}\right\|_{L^{\infty}} \leq \delta
$$

Hence,

$$
\left|\sigma-\frac{\partial \xi_{1}}{\partial x}-\frac{\partial h}{\partial x}\right|=\left|\dot{R}_{0}-\frac{\partial h}{\partial x}\right| \geq\left|\sigma\left(l_{0}\right)-\frac{\partial h}{\partial x}\right| .
$$

With this observation, the calculations in Lemma 2.1, starting from (2.36), where the equality sign should be replaced with " $\geq$ " remain unchanged, yielding

$$
\mathcal{E}(\xi+h)-\mathcal{E}(\xi) \geq 0
$$

for $h$ satisfying $\xi+h \in \mathcal{D}$ and (2.45). This proves that $\xi$ is a local minimizer, however, due to convexity of the integrand it is also a global one. Thus, indeed $(\Gamma, \xi)$ is a variational solution. Next, we may use Theorem 2.2 to deduce uniqueness.

The other case at first glance appears to be the worst to deal with. Because from (ii) and (2.41) we conclude that $\dot{l}_{0}<0$ and we cannot use formula (2.42) to determine $d\left(t, l_{0}(t)\right)$ and $d_{x}^{+}\left(t, l_{0}(t)\right)$. It seems that we need ad hoc assumptions to solve (2.41). However, before continuing let us change the notation and instead of $l_{0}$ let us write $\lambda_{0}$. Then, condition (ii) reads,

$$
f_{0}^{\lambda_{0}} \sigma(s) d s+\frac{\gamma\left(\mathbf{n}_{\Lambda}\right)}{\lambda_{0}}<\sigma\left(\lambda_{0}\right)
$$

Of course, due to our assumptions on $\sigma$ we can find $l_{0}<\lambda_{0}$ for which equality holds, i.e.

$$
\sigma\left(l_{0}\right)=f_{0}^{l_{0}} \sigma(s) d s+\frac{\gamma\left(\mathbf{n}_{\Lambda}\right)}{l_{0}} .
$$

If $\xi$ is a solution to (2.11), then it follows from Corollary 2.1 that $\frac{\partial \xi_{1}}{\partial x}\left(l_{0}\right)=0$. In other words, $\xi$ is a solution to an obstacle problem and $\xi$ on the interval $\left[l_{0}, \lambda_{0}\right]$ must be equal to the obstacle. Hence, $\left(-\lambda_{0}, \lambda_{0}\right)$ cannot be a inverse image of a faceted region, however, $\left(-l_{0}, l_{0}\right)$ is. The apparent case (ii) of violation of the tangency conditions turns out to 
be case (b) of Proposition 2.3, which yields legitimate data for (2.22) and subsequently a variational solution.

Let us make the final comment, if (ii) occurs for some $\xi \in \mathcal{D}$, satisfying $\dot{R}_{0}=\sigma-\operatorname{div}_{S} \xi$, then it may not be a solution to (2.11), and indeed an ad hoc assumption is necessary to solve (2.41), e.g. setting $d_{x}\left(t, l_{0}(t)\right)=d_{x}\left(0, l_{00}\right)$ gives a solutions. However, system (2.22) was derived from (2.16) for variational solutions and we do not have any chance for solving (2.41) in a systematic way.

\section{Evolution of a deformed rectangle in the plane}

Once we solved the graph case we may turn our attention to polygons evolving according to (1.1). There are two main reasons for focusing on rectangles only (or rather deformed rectangles, see Fig. 2 for possible evolution).

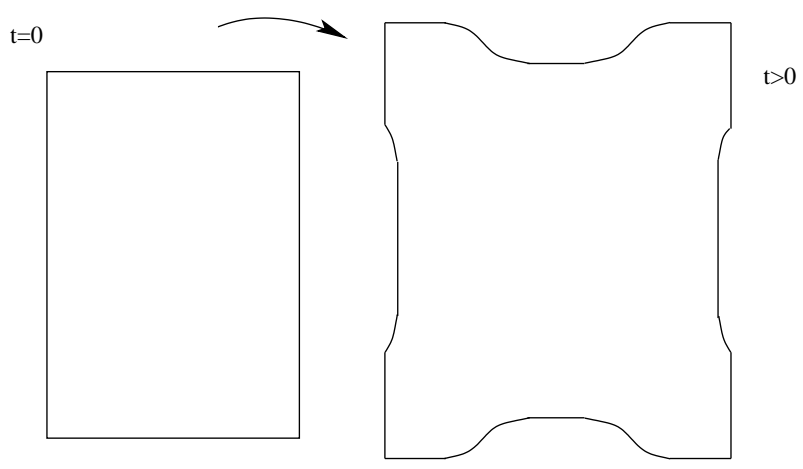

Fig. 2

First, they play a special role in our considerations because the problems of evolution of a straight circular cylinder reduces to evolution of a rectangle, (see [GR1], [GR3]). This problem is to be addressed in a separate paper. Second, in this case we can temporarily avoid the discussion of the proper notion of solution. One of the difficulties is of course defining the evolution of the corner. Fortunately, as we shall see the most important phenomena take place away from corners.

We want to use a notion of solution extending the one used in $\S 2$ as well as the notion of solution to a crystalline flow (see [Ta2], [AG]). The point is, the position of a corner $v$ is defined by the position of facets intersecting at $v$. For this approach proper assumptions on $\beta$ are necessary, this is why we keep (1.5) for $\gamma$ defined by (1.4). This in particular implies that the Wulff shape $W_{\gamma}$ of $\gamma$ is a rectangle.

\subsection{Setting up the problem}

Before stating the definition of solutions to (1.1) we will describe conditions on $\sigma$ which are more general than in $\S 1$. They will be consistent with properties deduced for $\sigma$ being a solution to the Laplace equation (see [GR1], [GR2]). Namely, we assume that $\sigma: \mathbb{R}_{+} \times$ $\mathbb{R}^{2} \rightarrow \mathbb{R}$ is continuous. Moreover, for all $t \geq 0, \sigma(t, \cdot, \cdot) \in C^{1}\left(\mathbb{R}^{2}\right)$ and the main exposition is concerned with $\sigma$ having no further smoothness in $t$. 
At the moment we consider $\sigma$ having (for fixed $t \geq 0$ ) a center of symmetry at the origin, i.e.

$$
\sigma(t,-x,-y)=\sigma(t, x, y) .
$$

Furthermore, for the sake of convenience and simplicity we assume

$$
\begin{aligned}
& \sigma\left(t,-x_{1}, x_{2}\right)=\sigma\left(t, x_{1}, x_{2}\right), \\
& \sigma\left(t, x_{1},-x_{2}\right)=\sigma\left(t, x_{1}, x_{2}\right) .
\end{aligned}
$$

Possible particular examples of such $\sigma$ are

$$
\begin{aligned}
& \sigma_{1}(t, x, y)=K(t)-\frac{1}{x^{2}+y^{2}+1}, \quad \sigma_{2}(t, x, y)=a(t) \ln \left(x^{2}+y^{2}+1\right) \\
& \sigma_{3}(t, x, y)=K(t)-\frac{1}{x^{2}+1}-\frac{1}{y^{2}+1} .
\end{aligned}
$$

Our goal is to study the bending of rectangles having normals belonging to the set of normals to the Wulff shape $W_{\gamma}$ (i.e. another fixed rectangle). Thus, we shall call $\Gamma$ a bent rectangle if the following condition is satisfied:

there exist even, Lipschitz continuous functions $d^{R}, d^{\Lambda}: \mathbb{R} \rightarrow \mathbb{R}_{+}$and there are positive numbers $L_{1}, R_{1}$ such that

$$
d^{\Lambda}\left(L_{1}\right)=R_{1} \quad d^{R}\left(R_{1}\right)=L_{1} .
$$

and

$$
\Gamma=\partial\left\{\left(x_{1}, x_{2}\right):\left|x_{1}\right| \leq d^{\Lambda}\left(x_{2}\right),\left|x_{2}\right| \leq d^{R}\left(x_{1}\right)\right\} .
$$

We shall call the points $\left( \pm d^{\Lambda}\left(x_{2}\right), \pm d^{R}\left(x_{1}\right)\right)$ vertexes of $\Gamma$. Thus, after we set

$$
\begin{aligned}
& S_{R}^{ \pm}=\left\{\left(x_{1}, x_{2}\right) \in \Gamma: x_{1}= \pm d^{\Lambda}\left(x_{2}\right), x_{2} \in\left[-R_{1}, R_{1}\right]\right\}, \\
& S_{\Lambda}^{ \pm}=\left\{\left(x_{1}, x_{2}\right) \in \Gamma: x_{2}= \pm d^{R}\left(x_{1}\right), x_{1} \in\left[-L_{1}, L_{1}\right]\right\}
\end{aligned}
$$

we notice that the graphs of $\pm d^{\Lambda}, \pm d^{R}$ make up the whole $\Gamma(t)$, i.e.

$$
\Gamma=S_{R}^{-} \cup S_{R}^{+} \cup S_{\Lambda}^{-} \cup S_{\Lambda}^{+} .
$$

We will collectively write $S_{R}$ for $S_{R}^{ \pm}$and $S_{\Lambda}$ for $S_{\Lambda}^{ \pm}$. We will call them facets of $\Gamma(t)$. Vertexes of $\Gamma$ are the intersections $S_{R}^{ \pm} \cap S_{\Lambda}^{ \pm}$. Moreover, the facets meet at vertexes at the right angle.

The following discussion parallel that of subsection 2.1. Some definitions may be repeated without changes while other need some adjustments. When possible we will refer to proper paragraph or subsection in $\S 2$ for the necessary notions. In fact, the definition are the same, what changes is the meaning of $\Gamma$.

The prime example is the Cahn-Hoffman vector $\xi$, (see paragraph 2.1.1) i.e. a vector field $\xi(t, \cdot): \Gamma \rightarrow \mathbb{R}^{3}$, such that $\left.\xi(t, \cdot)\right|_{S_{i}} \in \mathcal{D}_{i}, i=R, \Lambda$, where

$$
\begin{aligned}
& \mathcal{D}_{\Lambda}=\left\{\xi \in L^{\infty}\left(S_{\Lambda}\right): \xi(x) \in \partial \gamma(\mathbf{n}(x)), \operatorname{div}_{S} \xi \in L^{2}\left(S_{\Lambda}\right),(3.2) \text { holds }\right\}, \\
& \mathcal{D}_{R}=\left\{\xi \in L^{\infty}\left(S_{R}\right): \xi(x) \in \partial \gamma(\mathbf{n}(x)), \operatorname{div}_{S} \xi \in L^{2}\left(S_{R}\right),(3.2) \text { holds }\right\},
\end{aligned}
$$


where

$$
\xi\left( \pm L_{1}, \pm R_{1}\right) \in \partial \gamma\left( \pm \mathbf{n}_{\Lambda}\right) \cap \partial \gamma\left( \pm \mathbf{n}_{R}\right)
$$

On each facet we consider a minimization problem similar to (2.9). Namely, we set

$$
\mathcal{E}_{j}(\xi)=\frac{1}{2} \int_{S_{j}}\left|\sigma-\operatorname{div}_{S} \xi\right|^{2} d \mathcal{H}^{1}, \quad j=R, \Lambda .
$$

Now, we may explain our notion of solutions. After paragraph 2.1.1, by a solution to (1.1) we shall call a family of couples $(\Gamma(t), \xi(t)), t \in[0, T)$, where

(a) for each $t \geq 0$ the curve $\Gamma(t)$ is a bent rectangle and $d^{\Lambda}, d^{R}$ are continuous functions of its arguments, for each $x, d^{j}(\cdot, x), j=\Lambda, R$ are Lipschitz continuous and for each $t \in[0, T)$ the functions $d^{j}(t, \cdot), j=\Lambda, R$ are Lipschitz continuous;

(b) $\xi: \bigcup_{t \in[0, T)}\{t\} \times \Gamma(t) \rightarrow \mathbb{R}^{2}$ is at each time instant a Cahn-Hoffman vector. If $M:=\sup _{t \in[0, T)} \max \left\{L_{1}(t), R_{1}(t)\right\}+1$, and if for $j=\Lambda, R$ we set

$$
\begin{gathered}
\tilde{\xi}^{R}(t, x)= \begin{cases}\left(-\gamma\left(\mathbf{n}_{\Lambda}\right), \gamma\left(\mathbf{n}_{R}\right)\right) & x \in[-M,-L(t)], \\
\xi\left(t,\left(x, d^{R}(t, x)\right)\right) & x \in[-L(t), L(t)], \\
\left(\gamma\left(\mathbf{n}_{\Lambda}\right), \gamma\left(\mathbf{n}_{R}\right)\right) & x \in[L(t), M] ;\end{cases} \\
\tilde{\xi}^{\Lambda}(t, x)= \begin{cases}\left(-\gamma\left(\mathbf{n}_{\Lambda}\right),-\gamma\left(\mathbf{n}_{R}\right)\right) & x \in[-M,-R(t)], \\
\xi\left(t,\left(d^{\Lambda}(t, x), x\right)\right) & x \in[-R(t), R(t)], \\
\left(-\gamma\left(\mathbf{n}_{\Lambda}\right), \gamma\left(\mathbf{n}_{R}\right)\right) & x \in[R(t), M] ;\end{cases}
\end{gathered}
$$

then we assume that $t \mapsto \tilde{\xi}^{j}(t, \cdot) \in L^{\infty}\left(0, T ; L^{2}(-M, M)\right), j=\Lambda, R$;

(c) Equation (1.1) is satisfied in the $L^{2}$ sense for a.e. $t \geq 0$.

We call a solution $(\Gamma, \xi)$ variational (see paragraph 2.1.2) if for each $\left.t \in[0, T) \xi\right|_{S_{j}}(t) \in$ $L^{2}\left(S_{j}\right)$ is a solution to

$$
\mathcal{E}_{j}(\xi)=\min \left\{\mathcal{E}_{j}(\zeta): \zeta \in \mathcal{D}_{j}\right\}, \quad j=R, \Lambda .
$$

According to our definition each of the functions $d^{j}(t, \cdot)$ satisfies (2.12). Obviously, flat facets play a special role in our considerations. The definition of a faceted region in paragraph 2.1.2 requires a minor change. Namely, the reference in (2.13) to solutions of (2.11) should be replaced by the reference to solutions of (3.4).

We will consider only $\Gamma(t)$ such that for each $t \geq 0$ every side of $\Gamma(t)$ has no more than three faceted regions and the vertexes of $\Gamma(t)$ belong to the closure of the faceted regions. For the sake of convenience, following (2.14) we introduce $\tilde{d}^{j}(t, x), j=\Lambda, R$, then we may repeat the definition of the inverse images of faceted regions.

Furthermore, we also need the following relation on $\sigma$,

$$
x_{i} \frac{\partial \sigma}{\partial x_{i}}\left(x_{1}, x_{2}\right)>0, \quad x \neq 0, i=1,2 .
$$

We are interested in the behavior of the system past the breaking/bending phenomenon. Thus the some restrictions as in the previous sections apply to $\xi$. Hence we may rewrite (1.1) in a fashion similar to (2.16), however, we have to make an assumption making the conclusion of Proposition 2.1 valid. Namely, let us suppose that $l_{i}^{j}, i=0,1, j=\Lambda, R$ are given throughout the solution to (3.4), then we set $E_{Z}^{j}=\left\{x \in\left(-l_{1}^{j},-l_{0}^{j}\right) \cup\left(l_{0}^{j}, l_{1}^{j}\right): \frac{\partial d^{j}}{\partial x}=\right.$ 
0 . We suppose that $d$ satisfy (2.12) and $\xi$ is a solution to (2.11) and finally (2.15) for each facet holds, i.e.

$$
\mathcal{H}\left(E_{Z} \backslash \text { int } E_{Z}^{j}\right)=0, \quad j=R, \Lambda .
$$

With all these assumptions we may repeat the work of $\S 2$ and come to the system

$$
\begin{array}{ccc}
\sigma-\frac{\partial \xi_{1}}{\partial x_{1}}=\beta\left(\mathbf{n}_{\Lambda}\right) \dot{R}_{0} & \text { on } & {\left[0, l_{0}(t)\right]} \\
\sigma=\beta\left(d_{x_{1}}^{R}\right) \frac{d_{t}^{R}}{\sqrt{1+\left(d_{x_{1}}^{R}\right)^{2}}} & \text { on } & {\left[l_{0}(t), l_{1}(t)\right]} \\
\sigma-\frac{\partial \xi_{1}}{\partial x_{1}}=\beta\left(\mathbf{n}_{\Lambda}\right) \dot{R}_{1} & \text { on } & {\left[l_{1}(t), L_{1}(t)\right]} \\
\sigma-\frac{\partial \xi_{2}}{\partial x_{2}}=\beta\left(\mathbf{n}_{R}\right) \dot{L}_{0} & \text { on } & {\left[0, r_{0}(t)\right]} \\
\sigma=\beta\left(d_{x_{2}}^{\Lambda}\right) \frac{d_{t}^{\Lambda}}{\sqrt{1+\left(d_{x_{2}}^{\Lambda}\right)^{2}}} & \text { on } & {\left[r_{0}(t), r_{1}(t)\right]} \\
\sigma-\frac{\partial \xi_{2}}{\partial x_{2}}=\beta\left(\mathbf{n}_{R}\right) \dot{L}_{1} & \text { on } & {\left[r_{1}(t), R_{1}(t)\right],}
\end{array}
$$

where $\mathbf{n}_{R}=(1,0), \mathbf{n}_{\Lambda}=(0,1)$. Here the meaning of $l_{0}, l_{1}, R_{0}, R_{1}$ is the same as in the previous section, $d^{R}$ plays the role of $d$ from the previous section and the definitions of $r_{0}, r_{1}, L_{0}, L_{1}, d^{\Lambda}$ corresponds to, respectively, the definitions of $l_{0}, l_{1}, R_{0}, R_{1}, d^{R}$.

We augment system (3.7) with initial data

$$
\begin{array}{r}
l_{0}(0)=l_{00}, \quad l_{1}(0)=l_{10}, \quad r_{0}(0)=r_{00}, \quad r_{1}(0)=r_{10} \\
R_{0}(0)=R_{00}, \quad R_{1}(0)=R_{10}, \quad L_{0}(0)=L_{00}, \quad L_{1}(0)=L_{10} \\
d^{R}\left(0, x_{1}\right)=d_{0}^{R}\left(x_{1}\right), \quad d^{\Lambda}\left(0, x_{2}\right)=d_{0}^{\Lambda}\left(x_{2}\right)
\end{array}
$$

We assume that the inverse images of faceted regions contained in $S_{\Lambda}$ (resp. $S_{R}$ ) are $\left(-L_{1},-l_{1}\right),\left(-l_{0}, l_{0}\right),\left(l_{0}, L_{1}\right)$ (resp. $\left(-R_{1},-r_{1}\right),\left(-r_{0}, r_{0}\right)$ and $\left.\left(r_{1}, R_{1}\right)\right)$. We take into account the matching conditions, they take the form

$$
d_{0}^{R}\left(l_{00}\right)=R_{00}, \quad d_{0}^{R}\left(l_{10}\right)=R_{10} \quad d_{0}^{\Lambda}\left(r_{00}\right)=L_{00}, \quad d_{0}^{\Lambda}\left(r_{10}\right)=L_{10} .
$$

We also consider tangency conditions on $S_{\Lambda}$ and $S_{R}$, definition (2.19) requires no changes. After we assume (3.6) we may apply Proposition 2.1 to deduce (see paragraph 2.1.2) the form of the tangency conditions similar to (2.30), in the present case we obtain the relations,

$$
\begin{aligned}
\sigma\left(0, l_{00}, R_{00}\right) & =f_{0}^{l_{00}} \sigma\left(0, s, R_{00}\right) d s+\frac{\gamma\left(\mathbf{n}_{\Lambda}\right)}{l_{00}}, \\
\sigma\left(0, l_{10}, R_{10}\right) & =\int_{l_{10}}^{L_{10}} \sigma\left(0, s, R_{10}\right) d s-\frac{2 \gamma\left(\mathbf{n}_{\Lambda}\right)}{L_{10}-l_{10}}, \quad \xi_{1}\left(l_{i}\right)=-\gamma\left(\mathbf{n}_{\Lambda}\right) \quad i=0,1 \\
\sigma\left(0, L_{00}, r_{00}\right) & =\int_{0}^{r_{00}} \sigma\left(0, L_{00}, s\right) d s+\frac{\gamma\left(\mathbf{n}_{R}\right)}{r_{00}}, \\
\sigma\left(0, L_{10}, r_{10}\right) & =\int_{r_{10}}^{R_{10}} \sigma\left(0, L_{10}, s\right) d s-\frac{2 \gamma\left(\mathbf{n}_{R}\right)}{R_{10}-r_{10}}, \quad \xi_{2}\left(r_{i}\right)=-\gamma\left(\mathbf{n}_{R}\right) \quad i=0,1
\end{aligned}
$$


If we are given a solution to (1.1), whose each facet has at most three facetted regions with the maximality property (see paragraph 2.1.4), then after writing (3.7) in terms of $d^{R}, d^{\Lambda}, R_{0}, R_{1}, L_{0}, L_{1}$ and using the simplifying assumption (1.5), we come to the system

$$
\begin{aligned}
& \dot{R}_{0}=f_{0}^{l_{0}} \sigma\left(t, s, R_{0}\right) d s+\frac{\gamma\left(\mathbf{n}_{\Lambda}\right)}{l_{0}} \quad \text { on } \quad\left[0, l_{0}\right] \\
& \frac{\partial}{\partial t} d^{R}=\sigma\left(t, x_{1}, d^{R}\right) \quad \text { on } \quad\left[l_{0}, l_{1}\right] \\
& \dot{R}_{1}=f_{l_{1}}^{L_{1}} \sigma\left(t, s, R_{1}\right) d s-\frac{2 \gamma\left(\mathbf{n}_{\Lambda}\right)}{L_{1}-l_{1}} \quad \text { on } \quad\left[l_{1}, L_{1}\right] \\
& \dot{L}_{0}=\int_{0}^{r_{0}} \sigma\left(t, L_{0}, s\right) d s+\frac{\gamma\left(\mathbf{n}_{\Lambda}\right)}{r_{0}} \quad \text { on } \quad\left[0, r_{0}\right] \\
& \frac{\partial}{\partial t} d^{\Lambda}=\sigma\left(t, d^{\Lambda}, x_{2}\right) \quad \text { on } \quad\left[r_{0}, r_{1}\right] \\
& \dot{L}_{1}=\int_{r_{1}}^{R_{1}} \sigma\left(t, L_{1}, s\right) d s-\frac{2 \gamma\left(\mathbf{n}_{R}\right)}{R_{1}-r_{1}} \quad \text { on } \quad\left[r_{1}, R_{1}\right]
\end{aligned}
$$

augmented with initial conditions (3.8). We stress that for simplicity of notation the time variable $t$ was suppressed in (3.11) (except for function $\sigma$ ).

We explicitly assume here that

$$
r_{00} \leq r_{10}, \quad l_{00} \leq l_{10} .
$$

It is apparent from (3.11) that this system is solvable if we know beforehand the functions of time $l_{0}, l_{1}, r_{0}, r_{1}$. By Proposition 2.5 we conclude that $l_{0}=l_{0}\left(t, R_{0}\right), l_{1}=l_{1}\left(t, R_{1}, L_{1}\right)$, $r_{0}=r_{0}\left(t, L_{0}\right), r_{1}=r_{1}\left(t, L_{1}, R_{1}\right)$ are uniquely defined $C^{1}$ functions of their arguments. There is no reason to expect that

$$
r_{i}(t) \equiv r_{i 0}, \quad l_{i}(t) \equiv l_{i 0}, \quad i=0,1 .
$$

Thus, we have to make sure that

$$
\begin{aligned}
& r_{0}(t) \leq r_{00} \leq r_{10} \leq r_{1}(t), \quad l_{0}(t) \leq l_{00} \leq l_{10} \leq l_{1}(t), \\
& r_{0}, l_{0} \text { are decreasing } \quad r_{1}, l_{1} \text { are increasing. }
\end{aligned}
$$

We cannot at this point present general assumptions on $\sigma$ guaranteeing that (3.12) holds. But (3.12) is critical for the further development, that is why we shall say that the working hypothesis is satisfied if (3.12) is fulfilled. We present a simple example of $\sigma$ below.

Proposition 3.1 If $\sigma=\sigma_{3}$ is given by formula (3.1), where $K$ is smooth, then

$$
l_{0}(t) \equiv l_{00}, \quad i_{1}(t)>0, \quad r_{0}(t) \equiv r_{00}, \quad \dot{r}_{1}(t)>0 .
$$

Proof. It is sufficient to consider only $l_{i}, i=0,1$. We start with $l_{0}$. We differentiate the tangency conditions

$$
l_{0} \sigma\left(t, l_{0}, R_{0}\right)=\int_{0}^{l_{0}} \sigma\left(t, s, R_{0}\right) d s+\gamma\left(\mathbf{n}_{\Lambda}\right)
$$


with respect to time. This yields

$$
l_{0} \dot{l}_{0} \frac{\partial \sigma}{\partial x_{1}}\left(t, l_{0}, R_{0}\right)+l_{0} \frac{\partial \sigma}{\partial x_{2}}\left(t, l_{0}, R_{0}\right)=\int_{0}^{l_{0}} \frac{\partial \sigma}{\partial x_{2}}\left(t, s, R_{0}\right) d s .
$$

It is now obvious that in order to proceed we have to be able to compare $\frac{\partial \sigma}{\partial x_{2}}$ evaluated at $\left(t, l_{0}, R_{0}\right)$ with its average over $\left[0, l_{0}\right]$, e.g. monotonicity of the derivative $\frac{\partial \sigma}{\partial x_{2}}$ would help. In our case these two quantities are simply equal, hence

$$
l_{0} \dot{l}_{0} \frac{\partial \sigma}{\partial x_{1}}\left(t, l_{0}, R_{0}\right)=0 .
$$

The first claim follows.

In order to establish $\dot{l}_{1}(t)>0$ we differentiate the tangency conditions

$$
\left(L_{1}-l_{1}\right) \sigma\left(t, l_{1}, R_{1}\right)=\int_{l_{1}}^{L_{1}} \sigma\left(t, s, R_{1}\right) d s-2 \gamma\left(\mathbf{n}_{\Lambda}\right)
$$

with respect to time. This time the structure of $\sigma$ yields

$$
\begin{aligned}
& \frac{\partial \sigma}{\partial x_{1}}\left(t, l_{1}, R_{1}\right)\left(L_{1}-l_{1}\right) \dot{l}_{1}(t)+\frac{\partial \sigma}{\partial x_{2}}\left(t, l_{1}, R_{1}\right)\left(L_{1}-l_{1}\right) \dot{R}_{1}(t) \\
= & {\left[\sigma\left(t, L_{1}, R_{1}\right)-\sigma\left(t, l_{1}, R_{1}\right)\right] \dot{L}_{1}+\dot{R}_{1}(t) \int_{l_{1}}^{L_{1}} \frac{\partial \sigma}{\partial x_{2}}\left(t, s, R_{1}\right) d s . }
\end{aligned}
$$

We know that $\dot{L}_{1}>0$ see $\S 2$ and $s \mapsto \sigma\left(t, s, R_{1}\right)$ is strictly increasing. We have to be able to compare $\frac{\partial \sigma}{\partial x_{2}}$ evaluated at $\left(t, l_{1}, R_{0}\right)$ with its average over $\left[l_{1}, L_{1}\right]$. For this purpose we use the present assumption on $\sigma$, which guarantees that they are equal and cancel out. Thus, we deduce the claim.

Remark 3.1 It is not clear how to show that the working hypotheses is satisfied for $\sigma=\sigma_{1}$ or more generally, $\sigma=K(t)-f\left(x^{2}+y^{2}\right)$ for a strictly decreasing function $f$ without stringent assumption on $f$. While it is easy to see that for such $\sigma$ it is true that $i_{0}<0$ it is not obvious how to deduce $\dot{l}_{1} \geq 0$.

Now, we have to investigate the influence of the working hypothesis on validity the matching condition. Since $l_{0}(t)$ decreases and $l_{1}(t)$ increases, the faceted region $\left(-l_{0}, l_{0}\right)$ is being over taken by the curved one. Thus if $x_{0}=l_{0}(t), t>0$, then the characteristic starting at $t=0$ in $x_{0}$, i.e. $\tau \mapsto\left(x_{0}, \tau\right)$ will terminate at time $t$ and the value of $R_{0}(t)$ is the new initial data for $d^{R}$,

$$
d^{R}\left(t, l_{0}(t)\right):=R_{0}(t), \quad t>0 .
$$

A similar reasoning is valid for $l_{1}$ and $r_{i}, i=0,1$.

Let us notice that, time continuity (resp. differentiability) of $\sigma$ implies that $l_{i}, i=0,1$ are continuous (resp. differentiable) functions of $t$. It turns out that smoothness of $l_{i}$ yields propagation of smoothness of $d^{R}$ at $l_{i}(t)$. Namely, we have the following result.

Proposition 3.2 Let us suppose that $(\Gamma(t), \xi(t)), t \in[0, T)$ is a solution to (1.1), whose facets satisfy the matching condition and $\sigma$ is of class $C^{1}$. We assume that (3.13) holds. 
(a) If $\dot{l}_{0}<0$ (resp. $\dot{r}_{0}<0$ ), then

$$
\frac{\partial d^{R}}{\partial x}\left(t, l_{0}(t)\right)=0 \quad\left(\text { resp. } \frac{\partial d^{\Lambda}}{\partial x}\left(t, r_{0}(t)\right)=0\right)
$$

(b) If $\dot{l}_{1}>0$ (resp. $\left.\dot{r}_{1}>0\right)$, then

$$
\frac{\partial d^{R}}{\partial x}\left(t, l_{1}(t)\right)=0 \quad\left(\text { resp. } \frac{\partial d^{\Lambda}}{\partial x}\left(t, r_{1}(t)\right)=0\right)
$$

Proof. If is sufficient to show (a) for $l_{0}$ since the remaining cases are similar. We will show that

$$
\left.\frac{\partial d^{R}}{\partial x_{1}}\left(t, x_{1}\right)\right|_{x_{1}=l_{0}(t)}=0 \text {. }
$$

We consider the difference quotients for $h>0$ and $h<0$ separately. Namely, if $h<0$, then

$$
\frac{1}{h}\left(d^{R}\left(t, l_{0}(t)+h\right)-d^{R}\left(t, l_{0}(t)\right)\right)=R_{0}(t)-R_{0}(t)=0 .
$$

Hence, the left derivative of $d^{\Lambda}$ at $x=l_{0}(t)$ is zero.

Now, we take $h>0$. Because of the working hypothesis there exists a unique $\tau(h)$ such that

$$
l_{0}(t)+h=l_{0}(\tau(h)) .
$$

After little algebra we can see that $h \mapsto \tau(h)$ is differentiable and

$$
1=\left.i_{0}(t) \cdot \frac{d \tau(h)}{d} h\right|_{h=0}
$$

Hence,

$$
\begin{aligned}
\left.d^{R}\left(t, l_{0}(t)+h\right)-d^{R}\left(t, l_{0}(t)\right)\right) & =d^{R}\left(t, l_{0}(\tau(h))\right)-R_{0}(t) \\
& =d^{R}\left(t, l_{0}(\tau(h))\right)-d^{R}(\tau(h), \tau(h))+R_{0}(\tau(h))-R_{0}(t) \\
& =\int_{\tau(h)}^{t} d_{t}^{R}\left(s, l_{0}(\tau(h))\right) d s+R_{0}(\tau(h))-R_{0}(t) .
\end{aligned}
$$

After dividing by $h$ and passing to the limit we can see

$$
\lim _{h \rightarrow 0^{+}} \frac{1}{h}\left(d^{\Lambda}\left(t, l_{0}(t)+h\right)-d^{\Lambda}\left(t, l_{0}(t)\right)\right)=-\frac{d \tau}{d h}(0) \sigma\left(t, l_{0}(t), R_{0}(t)\right)+\dot{R}_{0}(t) \frac{d \tau}{d h}(0)=0
$$

The last equality follows from the tangency conditions.

This result means that the smoothness propagates, provided that $l_{0}$ is strictly decreasing. If $i_{0}\left(t_{0}\right)=0$, then (3.14) cannot be inferred at $t_{0}$. In other words, in any case the only possible scenario is that the curved part of $\Gamma(t)$ grows at the expense of the flat parts.

Moreover, we are now able to consider the degenerate case i.e.

$$
l_{00}=l_{10} .
$$




\subsection{Solvability of the ODE system}

Theorem 3.1 Let us consider system (3.11) augmented with initial data (3.8). We assume that:

(i) the data $d_{0}^{\Lambda}, d_{0}^{R}$ are sufficiently smooth, i.e. $d_{0}^{R} \in C^{1}\left(\left[l_{00}, l_{10}\right]\right), d_{0}^{\Lambda} \in C^{1}\left(\left[r_{00}, r_{10}\right]\right)$;

(ii) the matching conditions (3.9) are satisfied;

(iii) conditions (3.6) holds;

(iv) the tangency conditions (3.10) are fulfilled.

In addition the working hypothesis (3.12) is supposed to be valid due to an appropriate choice of $\sigma$ (e.g. $\sigma_{3}$ in (3.1) will do). Then there is variational solution to (1.1) starting at $t=0$ with a bent rectangle $\Gamma_{0}$, corresponding to $d_{0}^{\Lambda}, d_{0}^{R}$.

Remark 3.2. It is worth noting that the data $R_{00}=R_{10}$ and $l_{00}=l_{10}$ or $L_{00}=L_{10}$ and $r_{00}=r_{10}$ are permitted.

Proof. Let us recall that if the tangency condition hold, then by Proposition $2.5 l_{i}, i=0,1$ (resp. $\left.r_{i}, i=0,1\right)$ can be written as continuous functions, which are $C^{1}$ with respect to $R_{0}, R_{1}, L_{1}$, (resp. $L_{0}, L_{1}, R_{1}$ ). Hence (3.11) becomes a closed nonlinear system of ODE's for $L_{i}, R_{i}, i=0,1, d^{\Lambda}, d^{R}$. By the standard theory of ODE's it has a unique solution on a time interval $[0, T)$. Possibly, on a shorter interval the conditions $\left|d_{x}^{j}(t, x)\right| \leq 1, j=\Lambda, R$, hold.

In this system $x_{1}$ and $x_{2}$ play the role of parameters and $x_{1} \in\left[l_{00}, l_{10}\right], x_{2} \in\left[r_{00}, r_{10}\right]$. Due to the working hypothesis $l_{0}(\cdot)$ (resp. $\left.r_{0}(\cdot)\right)$ is decreasing while $l_{1}(\cdot)$ (resp. $r_{1}(\cdot)$ ) is increasing, as a result system (3.11) yield no value for $d^{R}(t, x)$ (resp. $d^{\Lambda}(t, x)$ ) for $t>0$ and $x \in\left[l_{0}(t), l_{00}\right] \cup\left[l_{10}, l_{1}(t)\right]$ (resp. $\left.x \in\left[r_{0}(t), r_{00}\right] \cup\left[r_{10}, r_{1}(t)\right]\right)$. However, the solution to (3.11) yields $l_{0}(t)=l_{0},\left(t, R_{0}(t)\right)$ etc.

In order to define $d^{R}\left(\tau, l_{i}(t)\right), \tau \geq t$ we consider the ODE's for $i=0,1$,

$$
\begin{aligned}
& d_{t}^{R}(\tau, x)=\sigma\left(\tau, x, d^{R}\right), \\
& d^{R}\left(t, l_{i}(t)\right)=R_{i}(t) .
\end{aligned}
$$

Similar problem should be considered for $d^{\Lambda}$, we omit the details. In this way we explicitly set the value of $d^{R}\left(t, l_{i}(t)\right)$. The above definition is correct because we assume that $l_{0}$ is decreasing and $l_{1}$ is increasing. Due to this definition the matching condition is satisfied automatically. Moreover, the tangency condition is fulfilled because of Proposition 2.5.

We notice that the solution we have just constructed satisfies (3.6). This is so, because due to the differential dependence of solutions to ODE's upon parameter we can conclude that for each $t>0$ functions $d^{R}(t, \cdot)\left(\right.$ resp. $\left.d^{\Lambda}(t, \cdot)\right)$ are $C^{1}$, possibly except at $l_{i}(t), i=$ 0,1 and $l_{i 0}$. However, we have shown that if $\dot{i}_{i}(t)$ has a sign, then $d^{R}(t, \cdot)$ is differentiable at $l_{i}(t)$. Thus, the exceptional set $E_{Z}^{\Lambda}$ may contain at most four points, $\pm l_{i 0}, i=0,1$, hence (3.6) is fulfilled.

Finally, we have to show that $\xi$ given by the formulas (2.28) is a solution to the minimization problem. We note that in this task $d^{\Lambda}$ and $d^{R}$ are fixed, hence we may use Lemma 2.1 again to conclude validity of our claim.

Remark 3.3 If $i_{0}<0, i_{1}>0$, then we can infer by Proposition 3.2 that smoothness propagates, i.e.

$$
\left.\frac{\partial d^{R}}{\partial x_{1}}\left(t, x_{1}\right)\right|_{x_{1}=l_{i}(t)}=0,\left.\quad \frac{\partial d^{\Lambda}}{\partial x_{2}}\left(t, x_{2}\right)\right|_{x_{2}=r_{i}(t)}=0 \quad i=0,1 .
$$


In order to prove uniqueness for the case of the deformed rectangle we will use methods applied in the proof of Theorem 2.2. However, the present task is more involved because of the lack of a transparent gradient flow structure. Moreover, we have to use more detailed information about the solutions we constructed as opposed to the graph case studied in the previous section. Thus, our result is less general than Theorem 2.2.

Theorem 3.2 Let us suppose that $\beta$ satisfies (1.5), $\left(\Gamma^{i}, \xi^{i}\right), i=1,2$ are two variational solutions to (1.1) defined on $[0, T)$ and $\Gamma^{1}(0, \cdot)=\Gamma^{2}(0, \cdot), \xi^{1}(0, \cdot)=\xi^{2}(0, \cdot)$. Moreover, the initial data satisfy the assumptions of Theorem 3.1. Then, $\Gamma^{1}(t, \cdot)=\Gamma^{2}(t, \cdot), \xi^{1}(t, \cdot)=$ $\xi^{2}(t, \cdot)$ for all $t \in[0, T)$.

Proof. One of the problems we have to overcome is the time dependence of domains of $d^{R_{i}}, d^{\Lambda_{i}}, i=1,2$. We have to extend them to fixed domains. We set $M=1+$ $\sup _{t<T} \max _{i=1,2}\left\{L_{1}^{i}(t), R_{1}^{i}(t)\right\}$. By assumption $\sigma$ is defined over $\mathbb{R}^{2}$. We will extend $d^{R_{i}}$, $d^{\Lambda_{i}}$ and $\xi^{i}, i=1,2$. However, in order to simplify the notation we will concentrate on $d^{R_{i}}, i=1,2$. The argument for $d^{\Lambda_{i}}$ is the same and will be omitted. First, we extend $\xi^{i}$ to $[-M, M]$ by formula (3.3). The extension of $d^{R_{i}}, i=1,2$ is by solution to the system

$$
\frac{\partial d^{R_{i}}}{\partial t}=\sigma-\frac{\partial \xi_{i}}{\partial x_{1}} \quad x \in[-M, M] \backslash\left[-L_{1}^{i}, L_{1}^{i}\right]
$$

We stress that $\sigma$ appearing in (3.15) is actually equal to $\sigma\left(t, x_{1}, d^{R_{i}}\left(t, x_{1}\right)\right)$. Subsequently, we proceed as in the proof of Theorem 2.2, i.e. we take the difference of (3.15) 1 for $i=1,2$, multiply by $d^{R_{2}}-d^{R_{1}}$ and integrate over $[-M, M]$. Hence, we obtain

$$
\begin{aligned}
\frac{1}{2} \frac{d}{d t} \int_{-M}^{M}\left|d^{R_{2}}-d^{R_{1}}\right|^{2} d x= & \int_{-M}^{M}\left[\sigma\left(t, x, d^{R_{2}}(t, x)\right)-\sigma\left(t, x, d^{R_{1}}(t, x)\right)\left(d^{R_{2}}-d^{R_{1}}\right) d x\right. \\
& \quad+\int_{-M}^{M}\left(-\frac{\partial \xi_{1}^{2}}{\partial x_{1}}+\frac{\partial \xi_{1}^{1}}{\partial x_{1}}\right)\left(d^{R_{2}}-d^{R_{1}}\right) d x \\
= & I+J .
\end{aligned}
$$

The first term is easily handled due to Lipschitz continuity of $\sigma$, we obtain

$$
I \leq C \int_{-M}^{M}\left|d^{R_{2}}-d^{R_{1}}\right|^{2} d x .
$$

In order to proceed we have to examine $\xi^{i}$ and to introduce some notation for that purpose. Namely, we shall write $\xi=\xi(R, L, \cdot)$ to denote the unique solution to (2.11) for $d=d^{R}$ defined over $[-L, L]$, in fact, as we have seen in the course of the proof of Theorem 2.1, it is a unique solution to the corresponding Euler-Lagrange equation. Hence in our case $\xi^{i}=\xi^{i}\left(R^{i}, L^{i}, \cdot\right), i=1,2$. Similar notation should be used for the Cahn-Hoffman vectors defined over the other pair of facets $S_{\Lambda}$, however, for the sake of simplicity of notation we shall not do this.

Using the new notation we will rewrite the term $J$, namely

$$
\begin{aligned}
J= & \int_{-M}^{M}\left(-\frac{\partial \xi_{1}}{\partial x_{1}}\left(R^{2}, L^{2}, x\right)+\frac{\partial \xi_{1}}{\partial x_{1}}\left(R^{1}, L^{2}, x\right)\right)\left(d^{R_{2}}-d^{R_{1}}\right)(x) d x \\
& \quad+\int_{-M}^{M}\left(-\frac{\partial \xi_{1}}{\partial x_{1}}\left(R^{1}, L^{2}, x\right)+\frac{\partial \xi_{1}}{\partial x_{1}}\left(R^{1}, L^{1}, x\right)\right)\left(d^{R_{2}}-d^{R_{1}}\right)(x) d x \\
= & J_{1}+J_{2} .
\end{aligned}
$$


An argument based on monotonicity of the operator $\partial \gamma$, as in the proof of Theorem 2.2, yields that $J_{1} \leq 0$. We turn our attention to $J_{2}$.

Vector fields $\xi\left(R^{1}, L^{i}\right), i=1,2$ are obtained as solutions to the Euler-Lagrange equation for the same $d^{R_{1}}$ but different $L_{1}^{i}$. Hence, by the definition of $l_{0}^{i}$ and the proof of Proposition $2.5 l_{0}^{1}=l_{0}^{2}$. As a result, by the formula for $\xi\left(R^{1}, L^{i}, x\right)$, on $\left(-l_{0}, l_{0}\right)$ we deduce

$$
\frac{\partial \xi_{1}}{\partial x_{1}}\left(R^{1}, L^{2}, x\right)=\frac{\partial \xi_{1}}{\partial x_{1}}\left(R^{1}, L^{1}, x\right) \quad \text { for } x \in\left(-l_{0}, l_{0}\right)
$$

However, the above argument is no longer valid for $l_{1}^{i}, i=1,2$, hence we obtain

$$
J_{2}=\left(\int_{-M}^{-l_{0}}+\int_{l_{0}}^{M}\right)\left(-\frac{\partial \xi_{1}}{\partial x_{1}}\left(R^{1}, L^{2}, x\right)+\frac{\partial \xi_{1}}{\partial x_{1}}\left(R^{1}, L^{1}, x\right)\right)\left(d^{R_{2}}-d^{R_{1}}\right)(x) d x .
$$

Let us introduce further notation

$$
l_{1}^{k}=\min \left\{l_{1}^{2}, l_{1}^{1}\right\}, \quad l_{1}^{m}=\max \left\{l_{1}^{2}, l_{1}^{1}\right\} \quad L_{1}^{i}=\min \left\{L_{1}^{2}, L_{1}^{1}\right\}, \quad L_{1}^{j}=\max \left\{L_{1}^{2}, L_{1}^{1}\right\} .
$$

Let us notice that

$$
\begin{array}{rlrl}
\frac{\partial \xi_{1}}{\partial x_{1}}\left(R^{1}, L^{p}\right)=0, & \text { for } x_{1} \in\left(l_{0}, l_{1}^{k}\right), \quad p=1,2, \\
\frac{\partial \xi_{1}}{\partial x_{1}}\left(R^{1}, L^{m}\right)=0, & \text { for } x_{1} \in\left(l_{1}^{k}, l_{1}^{m}\right), & \\
\frac{\partial \xi_{1}}{\partial x_{1}}\left(R^{1}, L^{i}\right)=0, & \text { for } x_{1} \in\left(L_{1}^{i}, L_{1}^{j}\right) .
\end{array}
$$

Hence,

$$
\begin{aligned}
J_{2} & \leq 2 \int_{l_{1}^{k}}^{l_{1}^{m}}\left|\frac{\partial \xi_{1}}{\partial x_{1}}\left(R^{2}, L^{k}\right)\right|\left|d^{R_{2}}-d^{R_{1}}\right| d x \\
& +2 \int_{l_{1}^{m}}^{L_{1}^{i}}\left|\frac{\partial \xi_{1}}{\partial x_{1}}\left(R^{2}, L^{2}\right)-\frac{\partial \xi_{1}}{\partial x_{1}}\left(R^{2}, L^{1}\right)\right|\left|d^{R_{2}}-d^{R_{1}}\right| d x \\
& +2 \int_{L_{1}^{i}}^{L_{1}^{j}}\left|\frac{\partial \xi_{1}}{\partial x_{1}}\left(R^{2}, L^{j}\right)\right|\left|d^{R_{2}}-d^{R_{1}}\right| d x
\end{aligned}
$$

The formulas for $\frac{\partial \xi_{1}}{\partial x_{1}}$, see (2.28), permit us to write

$$
J_{2} \leq K\left(\int_{l_{1}^{k}}^{l_{1}^{m}}\left|d^{R_{2}}-d^{R_{1}}\right| d x_{1}+\left|L_{1}^{2}-L_{1}^{1}\right|\left\|d^{R_{2}}-d^{R_{1}}\right\|_{L^{2}}+\left|L_{1}^{2}-L_{1}^{1}\right|\left|R_{1}^{2}-R_{1}^{1}\right|\right) .
$$

In order to reach the desired bound we have to show the following "reverse Hölder inequalities",

$$
\left|L_{1}^{2}-L_{1}^{1}\right| \leq C\left\|d^{\Lambda_{2}}-d^{\Lambda_{1}}\right\|_{L^{2}}, \quad\left|R_{1}^{2}-R_{1}^{1}\right| \leq C\left\|d^{R_{2}}-d^{R_{1}}\right\|_{L^{2}} .
$$

We will show them, possibly on after restricting the time intervals by the condition

$$
l_{1}^{i}(t) \leq l_{10}+a, \quad t \leq T_{1},
$$

for some $a>0$. Such $T_{1}$ exists because of continuity of $l_{1}^{i}$ (see Proposition 2.5). Thus, after recalling that $L_{1}^{i}(t) \geq L_{10}$, see Proposition 2.4,

$$
\left\|d^{R_{2}}-d^{R_{1}}\right\|_{L^{2}}^{2}=\int_{-M}^{M}\left|d^{R_{2}}-d^{R_{1}}\right|^{2} d x_{1} \geq \int_{l_{10}+a}^{L_{10}}\left|d^{R_{2}}-d^{R_{1}}\right|^{2} d x_{1}=\left|L_{10}-\left(l_{10}+a\right)\right|\left|R_{1}^{2}-R_{1}^{1}\right|^{2} .
$$


Hence, (3.16) follows.

In order to estimate that the remaining term $\int_{l_{1}^{k}}^{l_{1}^{m}}\left|d^{R_{2}}-d^{R_{1}}\right| d x_{1}$ we have to work a little bit more. We note that equations $(3.11)_{2}$ and (3.11) $)_{5}$ imply that $d^{R_{i}}, d^{\Lambda_{i}}, i=1,2$ are bounded on $\left[0, T_{1}\right] \times[-M, M]$ as long as

$$
\left\|d_{x}^{j}(0, \cdot)\right\|_{L^{\infty}} \leq L<\infty, \quad j=R, \Lambda .
$$

Using this information we arrive at

$$
J_{21}:=\int_{l_{1}^{k}}^{l_{1}^{m}}\left|d^{R_{2}}-d^{R_{1}}\right| d x_{1}=\int_{l_{1}^{k}}^{l_{1}^{m}}\left|R_{1}^{2}-R_{1}^{1}+\int_{x_{1}}^{l_{1}^{2}}\left(d_{x}^{R_{2}}-d_{x}^{R_{1}}\right) d s\right| d x_{1} .
$$

Hence

$$
J_{21} \leq\left|l_{1}^{2}-l_{1}^{1}\right|\left|R_{1}^{2}-R_{1}^{1}\right|+K\left|l_{1}^{2}-l_{1}^{1}\right|^{2} .
$$

We recall that by Proposition $2.5 l_{1}^{i}$ are of class $C^{1}$ and if $L_{1}^{2}=L_{1}^{1}$, then $l_{1}^{1}=l_{1}^{2}$. Thus

$$
\left|l_{1}^{2}-l_{1}^{1}\right| \leq K\left|L_{1}^{2}-L_{1}^{1}\right|
$$

Taking into account of above bounds we arrive at the estimate

$$
\frac{d}{d t}\left\|d^{R_{2}}-d^{R_{1}}\right\|_{L^{2}}^{2} \leq K\left(\left\|d^{R_{2}}-d^{R_{1}}\right\|_{L^{2}}^{2}+\left\|d^{\Lambda_{2}}-d^{\Lambda_{1}}\right\|_{L^{2}}^{2}\right) .
$$

A similar estimate is valid for the difference $d^{\Lambda_{2}}-d^{\Lambda_{1}}$, after adding them up we reach

$$
\frac{d}{d t}\left(\left\|d^{R_{2}}-d^{R_{1}}\right\|_{L^{2}}^{2}+\left\|d^{\Lambda_{2}}-d^{\Lambda_{1}}\right\|_{L^{2}}^{2}\right) \leq K\left(\left\|d^{R_{2}}-d^{R_{1}}\right\|_{L^{2}}^{2}+\left\|d^{\Lambda_{2}}-d^{\Lambda_{1}}\right\|_{L^{2}}^{2}\right) .
$$

Using Gronwall inequality we deduce that

$$
\left\|d^{R_{2}}-d^{R_{1}}\right\|_{L^{2}}^{2}+\left\|d^{\Lambda_{2}}-d^{\Lambda_{1}}\right\|_{L^{2}}^{2}=0
$$

for $t \in\left[0, T_{1}\right]$. Once we show that $\Gamma_{1}=\Gamma_{2}$, then $\xi^{1}=\xi^{2}$ follows from the strict convexity of the integrand in $\mathcal{E}_{i}, i=R, \Lambda$ as in the proof of Theorem 2.2.

\subsection{Final remarks}

If we encounter the data violating the tangency conditions, then their discussion parallels that on $\S 2.4$. However, some of the details has to be adjusted e.g. equation (2.43) become more involved, because of the time dependence of $\sigma$. Nonetheless, the main reasoning remains unchanged. As a result we may state the existence and uniqueness result for a case of violating the tangency conditions at $l_{00}$. The other ones are handles similarly.

Theorem 3.3 Let us suppose that all the assumptions of Theorem 3.1 hold, except the tangency condition $(2.40)_{1}$, but instead it the following inequality is satisfied

$$
\sigma\left(t, l_{0}(t)\right)-f_{0}^{l_{0}} \sigma(s) d s+\frac{\gamma\left(\mathbf{n}_{\Lambda}\right)}{l_{0}}<0 .
$$

Moreover, we assume that $d_{0}^{\Lambda} \in C^{1,1}\left(\left[l_{00}, l_{10}\right]\right)$ and $\sigma \in C^{1,1}$. Then there is a local in time variational solution to (1.1) and it is unique, however at no time instant $t>0$ condition the tangency condition is satisfied at $x_{1}=l_{0}(t)$. 
The proof goes along the lines of the proof of Theorem 2.3. Details are left to the interested Reader.

Finally, we would like to address the case of $\sigma$ decreasing along the facets, i.e. with reversed signs in (3.5),

$$
x_{i} \frac{\partial \sigma}{\partial x_{i}}\left(x_{1}, x_{2}\right)<0 \quad x \neq 0, i=1,2 .
$$

We notice that the notions of solution and variational solution do not depend upon particular behavior of $\sigma$, hence the derivation of (3.11) remains valid. The definition of matching and tangency condition is not affected either. Hence, if the initial data satisfy the tangency condition, then we could close system (3.11) by supplying $l_{i}, r_{i}, i=0,1$ constructed with the help of Proposition 2.5. The existence Theorem 3.1 requires only minor changes, at least if we keep the non-constant initial data for $d^{\Lambda}, d^{R}$ as in (2.12), the uniqueness Theorem 3.2 goes without any change at all.

Acknowledgment. The work of the first author was partly supported by a Grant-inAid for Scientific Research (no. 1534008) and by the formation of COE 'Mathematics of nonlinear structures via singularities' from the Japan Society of Promotion of Science. The second author was in part supported by KBN grant 1 P03A 03728 . Both authors enjoyed some support which was a result of Polish-Japanese Intergovernmental Agreement on Cooperation in the Field of Science and Technology.

\section{References}

[AG] S.Angenent, M.Gurtin, Multiphase thermomechanics interfacial structure. 2. Evolution of an isothermal interface, Arch. Rational Mech. Anal. 108, (1989), 323391.

[Ba] V.Barbu, "Nonlinear semigroups and differential equations in Banach spaces", Noordhoff, Leyden, 1976.

[BGN] G. Bellettini, R. Goglione, M. Novaga, Approximation to driven motion by crystalline curvature in two dimensions, Adv. Math. Sci. Apl. 10 (2000) 467-493.

[BNP1] Bellettini, G., Novaga, M., Paolini, M.: Characterization of facet breaking for nonsmooth mean curvature flow in the convex case, Interfaces and Free Boundaries, 3, 415-446 (2001)

[BNP2] Bellettini, G., Novaga, M., Paolini, M.: On a crystalline variational problem, part I: First variation and global $L^{\infty}$ regularity. Arch. Rational Mech. Anal., 157, 165191 (2001)

[BNP3] Bellettini, G., Novaga, M., Paolini, M.: On a crystalline variational problem, part II: $B V$ regularity and structure of minimizers on facets. Arch. Rational Mech. Anal., 157, 193-217 (2001)

[Br] H.Brezis, "Operateurs maximaux monotones et semi-group de contraction dans les espace de Hilbert", North Holland, Amsterdam, 1973.

[FG] Fukui,T., Giga, Y.: Motion of a graph by nonsmooth weighted curvature, in: World congress of nonlinear analysts '92, vol I, ed. V.Lakshmikantham, Walter de Gruyter, Berlin, 1996, 47-56 
[GG1] M.H. Giga, Y. Giga, A subdifferential interpretation of crystalline motion under nonuniform driving force. Dynamical systems and differential equations, vol. I (Springfield, MO, 1996). Discrete Contin. Dynam. Systems, Added Volume I, 276-287 (1998).

[GG2] M.-H. Giga and Y. Giga, Remarks on convergence of evolving graphs by nonlocal curvature, In : Progress in Partial Differential Equations vol. 1 (eds. H. Amann et al) Pitman Research Notes in Mathematics Series, 383 (1998), pp. 99-116, Longman, Essex, England.

[GG3] M.-H. Giga and Y. Giga, Generalized motion by nonlocal curvature in the plane, Arch. Rational. Mech. Anal., 159 (2001), 295-333.

[GG4] M.-H. Giga and Y. Giga, Minimal vertical singular diffusion preventing overturning for the Burges equation, Contem. Math., 330 (2003), 73-88.

[GG5] M.-H. Giga and Y. Giga, A PDE approach for motion of phase-boundaries by a singular interfacial energy, 'Stochastic analysis on large scale interacting systems' eds. T. Funaki etd pp.213-232. Adv. Studies in Pure Math. 39 (2004).

[GR1] Giga, Y., Rybka, P.: Quasi-static evolution of 3-D crystals grown from supersaturated vapor, Diff. Integral Eqs., 15, 1-15 (2002)

[GR2] Giga, Y., Rybka, P.: Berg's Effect. Adv. Math. Sci. Appl., 13, 625-637 (2003)

[GR3] Y. Giga, P. Rybka, Stability of facets of self-similar motion of a crystal, Adv. Diff. Eqs., 10 (2005), 601-634.

[GR4] Y. Giga, P. Rybka, Stability of facets of crystals growing from vapor, to appear in Dicrete and Cont. Dyn. Sys.

[KS] D.Kinderlehrer, G.Stampacchia, An Introduction to Variational Inequalities and their Applications, Academic Press, New York, 1980.

[N] L.Nirenberg, Topics in Nonlinear Functional Analysis, New York University, New York, 1974.

[R] P.Rybka, A crystalline motion: uniqueness and geometric properties, SIAM J. Appl. Math., 57 (1997), 53-72.

[Si] L.Simon, Lectures on Geometric Measure Theory. Proc. Centre for Math. Anal., Australian Nat. Univ. 3 (1983)

[St] A.Stancu, Uniqueness of Self-Similar Solutions for a Crystalline Flow, Indiana University Mathematics J., 45, (1996), pp. 1157-1174.

[Ta1] J.E.Taylor, Constructions and conjectures in crystalline nondifferential geometry, In: Differential Geometry (eds. B. Lawson and K. Tanenblat), Proceedings of the Conference on Differential Geometry, Rio de Janeiro, Pitman Monographs Surveys in Pure and Applied Math. 52 (1991) pp.321-336, Pitman, London

[Ta2] J.E.Taylor, Motion of curves by crystalline curvature, including triple junctions and boundary points, Proc. Symp. Pure Math., 54 (1993), pp. 417-438.

[Z] W.P.Ziemer, Weakly differentiable functions, Springer, New York, Berlin, 1989. 